\title{
Canadian
}

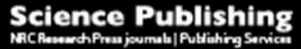

Canadian Journal of Physics

Revue canadienne de physique

\section{Natural Convection in a Triangular Cavity Filled with a Nanofluid-Saturated Porous Medium Using Three Heat Equation Model}

\begin{tabular}{|r|l|}
\hline Journal: & Canadian Journal of Physics \\
\hline Manuscript ID & cjp-2016-0053.R1 \\
\hline Manuscript Type: & Article \\
\hline Date Submitted by the Author: & 13 -Mar-2016 \\
\hline Complete List of Authors: & $\begin{array}{l}\text { Ghalambaz, Mohammad; Islamic Azad University of Dezful, Department of } \\
\text { Mechanical Engineering } \\
\text { Sabour, Mahmoud; Dezful Branch, Islamic Azad University, Dezful, Iran, } \\
\text { Department of Mechanical Engineering }\end{array}$ \\
\hline Keyword: & $\begin{array}{l}\text { Nanofluid-saturated porous media, Thermal non-equilibrium model, } \\
\text { Buongiorno model, Thermophoresis, Natural Convection }\end{array}$ \\
\hline
\end{tabular}

\section{SCHOLARONE" \\ Manuscripts}




\title{
Natural Convection in a Triangular Cavity Filled with a Nanofluid-Saturated Porous Medium Using Three Heat Equation Model
}

\author{
Mohammad Ghalambaz* \\ Department of Mechanical Engineering, Dezful Branch, Islamic Azad University, Dezful, Iran. \\ m.ghalambaz@gmail.com \\ Mahmoud Sabour \\ Department of Mechanical Engineering, Dezful Branch, Islamic Azad University, Dezful, Iran. \\ m.sabour1990@gmail.com
}

PACS: 44.30.+v Heat flow in porous media; 44.25.+f Natural convection;

iv *Corresponding author: Mohammad Ghalambaz, Assistant Professor at Mechanical Engineering is Department, Dezful Branch, Islamic Azad University, Dezful, Iran.

19 m.ghalambaz@iaud.ac.ir, Tell: +98 641 5261054, Fax: +98 6415263250. 
1

$r$

\section{Abstract}

The present study aims to examine the local thermal non-equilibrium natural convection heat and mass transfer of nanofluids in a triangular enclosure filled with a porous medium. The effect of the presence of nanoparticles and the thermal interaction between phases on the flow, temperature distribution of phases, the concentration distribution of nanoparticles as well as the Nusselt number of phases is theoretically studied. The interaction between the phases of nanoparticles and the base is taken into account by using a three thermal energy equation model while the concentration distribution of nanoparticles is modeled by the Buongiorno,s model. A hot flush element is mounted at the vertical wall of the triangle enclosure to provide constant temperature of $T_{h}$ while the inclined wall is at the constant temperature of $T_{c}$. A three heat equation model by considering local thermal non-equilibrium (LTNE) model of the nanoparticles, the porous medium and the base fluid is developed and utilized for natural convection of nanofluids in an enclosure. The drift-flux of nanoparticles due to the nano-scale effects of thermophoresis and Brownian motion effects is addressed. The governing equations are represented in a non-dimensional form and solved by employing the finite element method. The results indicate that the increase of Rayleigh number shows a significant increase in the average Nusselt number for the base fluid phase, a less significant increase in the average Nusselt number for the solid matrix phase, and almost an insignificant effect in the average Nusselt number of nanoparticles phase. The raise of the buoyancy ratio parameter (the ratio of mass transfer buoyancy forces to the thermal buoyancy forces) tends to reduce and increase the average Nusselt number in fluid and porous phases, respectively. An optimum value of buoyancy ratio parameter for the average Nusselt number of the nanoparticles phase is observed. 
1 Keywords: Nanofluid-saturated porous media; Thermal non-equilibrium model; Buongiorno model;

r Thermophoresis; Natural Convection.

\section{$\varepsilon \quad$ Nomenclature}

Latin Symbols

$A R \quad$ aspect ratio

C Nanoparticle volume fraction

$C_{0} \quad$ ambient nanoparticle volume fraction

$D_{B} \quad$ Brownian diffusion coefficient $\left(\mathrm{m}^{2} / \mathrm{s}\right)$

$D_{T} \quad$ thermophoretic diffusion coefficient $\left(\mathrm{m}^{2} / \mathrm{s}\right)$

g gravitational acceleration vector $\left(\mathrm{m} / \mathrm{s}^{2}\right)$

$h \quad$ height of heater $(m)$

$H \quad$ height of cavity $(m)$

$H_{H} \quad$ non dimensional Height of heater

$h_{f p} \quad$ interface heat transfer coefficients between the fluid/particle phases $\left(\mathrm{W} / \mathrm{m}^{3}{ }^{o} \mathrm{~K}\right)$

$h_{f s} \quad$ interface heat transfer coefficients between the fluid/solid-matrix phases $\left(\mathrm{W} / \mathrm{m}^{3}{ }^{\circ}{ }^{o} \mathrm{~K}\right)$

$K \quad$ permeability of the porous medium

$k \quad$ effective thermal conductivity $\left(W / m .{ }^{\circ} K\right)$

$L \quad$ length of cavity $(m)$

Le Lewis number

$\mathrm{Nb} \quad$ Brownian motion parameter

Nhp Nield number for the fluid/nanoparticle interface (fluid/nanoparticle interface parameter)

Nhs Nield number for the fluid/solid-matrix interface (fluid/solid-matrix interface parameter) 


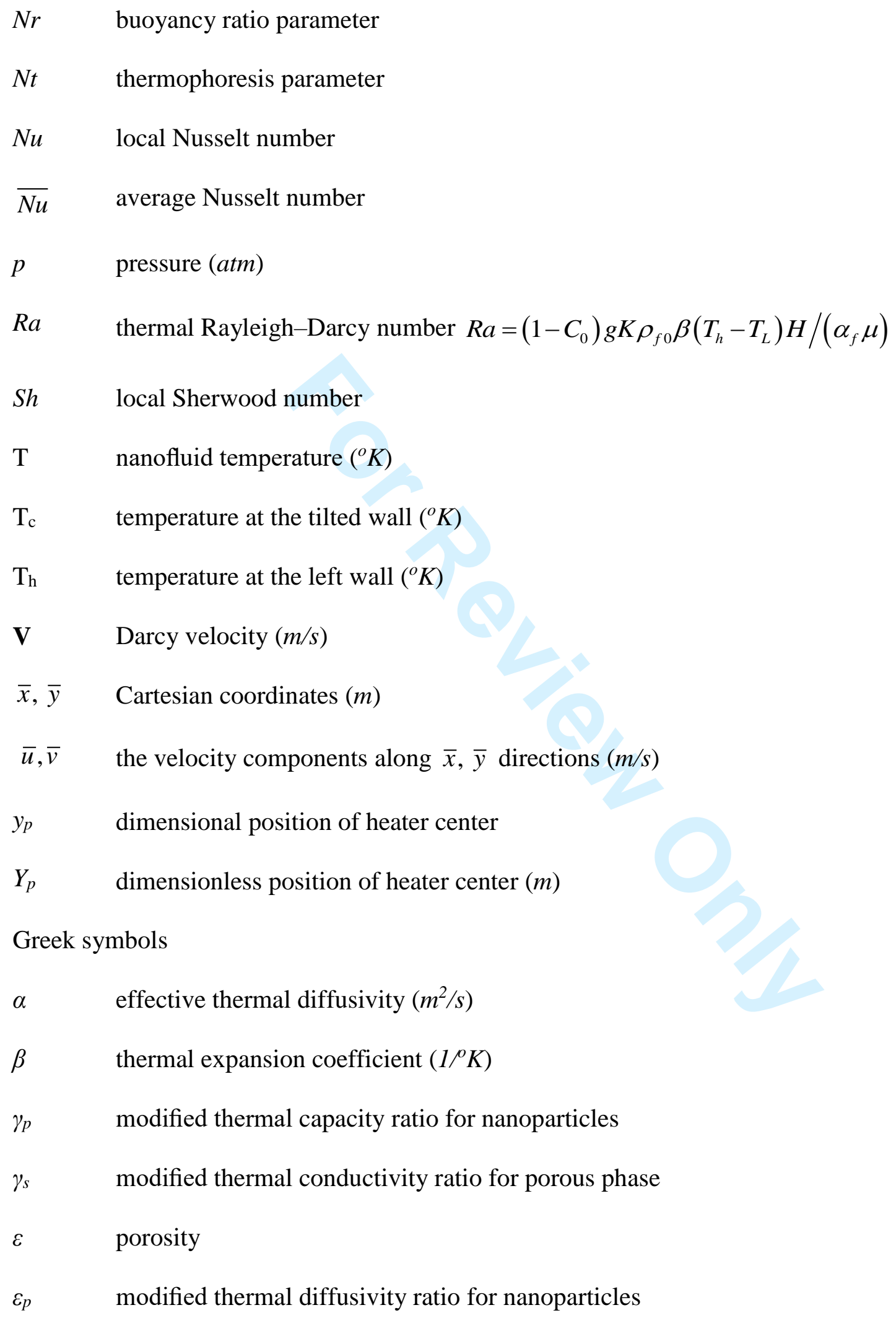




$\begin{array}{ll}\theta & \text { non-dimensional temperature } \\ \mu & \text { dynamic viscosity }(\mathrm{kg} / \mathrm{m} . \mathrm{s}) \\ \rho & \text { fluid density }\left(\mathrm{kg} / \mathrm{m}^{3}\right) \\ (\rho c) & \text { effective heat capacity }\left(\mathrm{J} / \mathrm{m}^{3} .{ }^{\circ} \mathrm{K}\right) \\ \tau & \text { parameter defined by } \tau=(\rho c)_{p} /(\rho \mathrm{c})_{f} \\ \psi & \text { non-dimensional stream function } \\ \bar{\psi} & \text { stream function }\left(\mathrm{m}^{2} / \mathrm{s}\right)\end{array}$

Subscripts

$0 \quad$ ambient property

$f \quad$ base fluid phase

$p \quad$ nanoparticle phase

$s \quad$ porous medium solid-matrix phase

\section{r 1. Introduction}

$r \quad$ The flow and heat transfer in porous media is subject of many industrial applications such as flow

$\varepsilon \quad$ through grains, fibers or compact heat exchangers. In many engineering and physical applications of

- convective heat transfer in porous media, it could be assumed that the interaction between the fluid and

1 the porous matrix is very high, and hence, the temperature difference between the flowing fluid and the

$\checkmark$ porous medium material is negligible. In this case, the energy of the fluid and porous medium can be

$\wedge$ represent by an affective heat equation for the mixture of the fluid and porous medium. This model is

$9 \quad$ known as local thermal equilibrium model.

1. There are many research studies in the literature, which have examined the free convective heat 1 transfer in porous enclosures, using the local thermal equilibrium model. For example, Baytas and Pop 
1

r

[1], Saeed and Pop [2], Basak et al. [3], Sathiyamoorthy et al. [4], Oztop et al. [5], Basak et al. [6], Chamkha and Ismael [7] have studied different aspect of the convective heat transfer in porous media utilizing the local thermal equilibrium model. However, there are many practical cases, in which the thermal equilibrium model between the phases is not valid, and the temperature of the phases is quite distinct. In these situations, the local thermal non-equilibrium models are required.

The local thermal non-equilibrium free convective heat transfer for regular fluid has found very important practical engineering applications in thermal removal systems and petroleum applications. For example, a highly conductive heat sink for cooling of high power electronic devices or the nuclear fuel rods in a cooling bath can be modeled by the local thermal non-equilibrium model of porous media. There are some excellent studies considering the local thermal non-equilibrium models for convective heat transfer of fluids in porous media [8-10].

Recently, nanofluids and high conductive metallic porous foams have been proposed as potential media to enhance the heat transfer for applications in heat removal systems. Nanofluids have been proposed as new engineered fluids with enhanced thermo-physical properties to increase the convective heat transfer potential of conventional heat transfer fluids [11-14]. Heat transfer potential of nanofluids has been examined in many recent studies [15-19]. In the case of metallic porous foams, as the thermal conductivity of porous foams is high, the temperature difference between the porous matrix and the free convective flow of the fluid could be important. Hence, in analysis of such systems, considering local thermal non-equilibrium models is very important.

The free convective heat transfer of nanofluids in enclosures, saturated with porous media, has been studied in some of the recent studies. For instance, Sun and Pop [20] have studied the free convective heat transfer of nanofluids in a triangular cavity by considering the local thermal equilibrium among the phases of nanoparticles, porous matrix and the base fluid. Sheremet et al. [21 and 22] have 
1

r.

r

r

rT

studied the convective heat transfer of nanofluids in a square cavity filled with a nanofluid by using Tiwari and Das' nanofluid model. Tiwari and Das' nanofluid model assumes a homogeneous distribution of nanoparticles in the base fluid and porous media. The Tiwari and Das' nanofluid model was also utilized by Ghalambaz et al. [23] to study the free convective heat transfer of nanofluids in a Parallelogrammic Porous Cavity filled with a porous medium.

Sheremet and Pop [24] have investigated the free convective heat transfer of nanofluids in a square cavity by using the Buongiorno's mathematical model [25]. The Buongiorno's mathematical model [25] evaluates the concentration distribution of nanoparticles due to the Brownian and thermophoresis effects. The effect of the presence of nanoparticles on the convective heat transfer was investigated for different temperature boundary conditions [24], and different geometries including shallow and slender porous cavities [26], and triangular porous cavity [27].

All of the mentioned studies in the literature for convective heat transfer of nanofluids in an enclosure filled with a porous medium have assumed local thermal equilibrium among the phases. The present study aims to examine the free convective heat transfer of nanofluids in a triangular enclosure by using the local thermal non-equilibrium model incorporating with Buongiorno's mathematical model [25]. The present study is the extension of the study of Sun and Pop [20] and Sheremet and Pop [27] for the case of local thermal non-equilibrium heat transfer of nanofluids.

\section{Basic equations}

Consider the steady state natural convection heat and mass transfer of nanofluids in a two-dimensional porous triangular cavity. The Cartesian coordinate system of $\bar{x}$ and $\bar{y}$ is adopted where $\bar{x}$ axis is aligned along the bottom wall, and $\bar{y}$ axis is aligned along the vertical wall. A schematic view of the coordinate system and problem modeling is depicted in Fig. 1. 
The height of the vertical wall of the triangle is $H$ while the length of the bottom horizontal wall is $L$. A heater with the height of $H_{H}$ and the center position of $Y_{p}$ is mounted at the left vertical wall. The heater holds the temperature of the wall at a constant temperature of $T_{h}$ while the remaining parts of the vertical wall are well insulated. The bottom wall is also well insulated while there is no heat or particles fluxes at the surface. The hypotenuse wall is cooled and maintained at the constant temperature of $T_{c}$. The flow in the porous medium is modeled using the Darcy-Boussinesq model. The local thermal nonequilibrium model is also employed to account the temperature difference between the phases. In the heat equations, the temperature difference between the base fluid and nanoparticles as well as the temperature difference between the base fluid and the solid-matrix is taken into account. Therefore, the thermal equations are described by a three temperatures model. It is assumed that the nanoparticles are well suspended in the nanofluid by using either surfactant or surface charge technology that prevents the nanoparticles from agglomeration and deposition on the porous matrix [28-31].

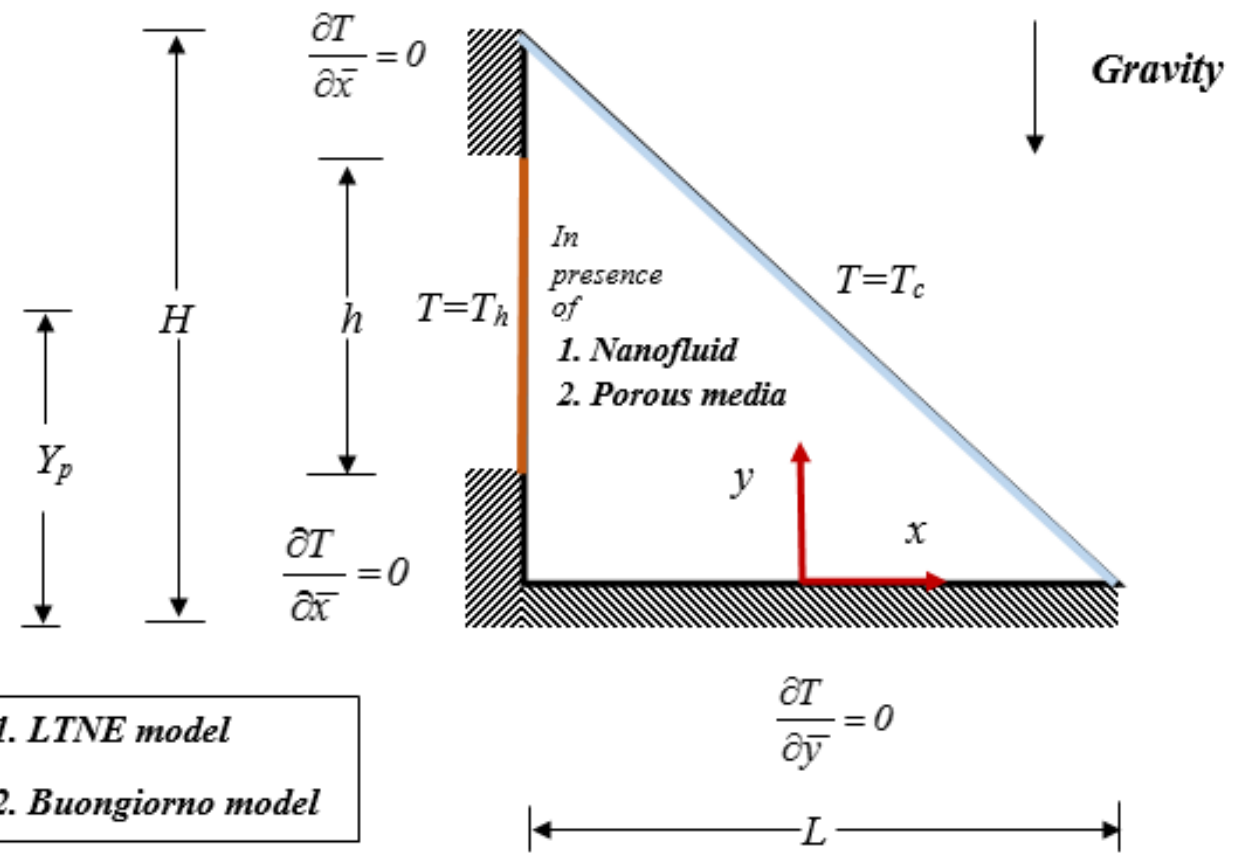

Fig. 1. Physical model and coordinate system. 
The conservation equations for the total mass of mixture, Darcy momentum for mixture, thermal $r \quad$ energy in the fluid phase, thermal energy in the particle phase, thermal energy in the solid-matrix phase, r and mass of nanoparticles are written as,

$\varepsilon \cdot \mathbf{V}=0$

- $\quad \frac{\mu}{K} \mathbf{V}=-\nabla p+\left[C \rho_{p}+(1-C) \rho_{f 0}\left(1-\beta\left(T_{f}-T_{c}\right)\right)\right] \mathbf{g}$

$1 \quad \frac{1}{\varepsilon} \mathbf{V} \cdot \nabla T_{f}=\frac{k_{f}}{(\rho c)_{f}} \nabla^{2} T_{f}+\tau\left(D_{B} \nabla C \cdot \nabla T_{f}+\frac{D_{T}}{T_{c}} \nabla T_{f} \cdot \nabla T_{f}\right)+\frac{\left[h_{f p}\left(T_{p}-T_{f}\right)+h_{f s}\left(T_{s}-T_{f}\right)\right]}{\varepsilon\left(1-C_{0}\right)(\rho c)_{f}}$

$\vee \quad \frac{1}{\varepsilon} \mathbf{V} \cdot \nabla T_{p}=\frac{k_{p}}{(\rho c)_{p}} \nabla^{2} T_{p}+\frac{h_{f p}}{\varepsilon C_{0}(\rho c)_{p}}\left(T_{f}-T_{p}\right)$

$\wedge \quad 0=\frac{k_{s}}{(\rho c)_{s}} \nabla^{2} T_{s}+\frac{h_{f s}}{(1-\varepsilon)(\rho c)_{s}}\left(T_{f}-T_{s}\right)$

$q \quad \frac{1}{\varepsilon} \mathbf{V} \cdot \nabla C=D_{B} \nabla^{2} C+\frac{D_{T}}{T_{c}} \nabla^{2} T_{f}$

11 where a detailed derivation of the above equations was proposed and discussed by Buongiorno [25],

ir Tzou [32 and 33], Nield and Kuznetsov [30], and Kuznetsov and Nield [28]. In the governing equations

ir for the conservation of thermal energy in the fluid phase (Eq. 3) as well as the mass conservation for

Is nanoparticles (Eq. 6), the Brownian transport and thermophoresis coefficients are assumed to be

10 constant [28 and 30] as the temperature differences in the system are assumed to be small. Now, by

17 introducing a stream function $\bar{\psi}$ defined by

iv $\quad \bar{u}=\frac{\partial \bar{\psi}}{\partial \bar{y}}, \quad \bar{v}=-\frac{\partial \bar{\psi}}{\partial \bar{x}}$ 
the continuity equation for the mixture, i.e. Eq. (1) is satisfied identically. The pressure could also be eliminated from the momentum Eq. (2) by cross differentiation. Hence, the remaining equations can be written as,

$\varepsilon \quad \frac{\partial^{2} \bar{\psi}}{\partial \bar{x}^{2}}+\frac{\partial^{2} \bar{\psi}}{\partial \bar{y}^{2}}=-\frac{\left(1-C_{0}\right) \rho_{f 0} g K \beta}{\mu} \frac{\partial T_{f}}{\partial \bar{x}}+\frac{\rho_{p}-\rho_{f 0}}{\mu} g K \frac{\partial C}{\partial \bar{x}}$,

$\frac{1}{\varepsilon}\left(\frac{\partial \bar{\psi}}{\partial \bar{y}} \frac{\partial T_{f}}{\partial \bar{x}}-\frac{\partial \bar{\psi}}{\partial \bar{x}} \frac{\partial T_{f}}{\partial \bar{y}}\right)=\alpha_{f}\left(\frac{\partial^{2} T_{f}}{\partial \bar{x}^{2}}+\frac{\partial^{2} T_{f}}{\partial \bar{y}^{2}}\right)+\tau\left\{D_{B}\left(\frac{\partial C}{\partial \bar{x}} \frac{\partial T_{f}}{\partial \bar{x}}+\frac{\partial C}{\partial \bar{y}} \frac{\partial T_{f}}{\partial \bar{y}}\right)+\right.$

$\left.+\left(\frac{D_{T}}{T_{c}}\right)\left[\left(\frac{\partial T_{f}}{\partial \bar{x}}\right)^{2}+\left(\frac{\partial T_{f}}{\partial \bar{y}}\right)^{2}\right]\right\}+\frac{\left[h_{f p}\left(T_{p}-T_{f}\right)+h_{f s}\left(T_{s}-T_{f}\right)\right]}{\varepsilon\left(1-C_{0}\right)(\rho c)_{f}}$

$1 \quad \frac{1}{\varepsilon}\left(\frac{\partial \bar{\psi}}{\partial \bar{y}} \frac{\partial T_{p}}{\partial \bar{x}}-\frac{\partial \bar{\psi}}{\partial \bar{x}} \frac{\partial T_{p}}{\partial \bar{y}}\right)=\alpha_{p}\left(\frac{\partial^{2} T_{p}}{\partial \bar{x}^{2}}+\frac{\partial^{2} T_{p}}{\partial \bar{y}^{2}}\right)+\frac{h_{f p}}{\varepsilon C_{0}(\rho c)_{p}}\left(T_{f}-T_{p}\right)$

$\vee \quad 0=\alpha_{s}\left(\frac{\partial^{2} T_{s}}{\partial \bar{x}^{2}}+\frac{\partial^{2} T_{s}}{\partial \bar{y}^{2}}\right)+\frac{h_{f s}}{(1-\varepsilon)(\rho c)_{s}}\left(T_{f}-T_{s}\right)$

$\wedge \quad \frac{1}{\varepsilon}\left(\frac{\partial \bar{\psi}}{\partial \bar{y}} \frac{\partial C}{\partial \bar{x}}-\frac{\partial \bar{\psi}}{\partial \bar{x}} \frac{\partial C}{\partial \bar{y}}\right)=D_{B}\left(\frac{\partial^{2} C}{\partial \bar{x}^{2}}+\frac{\partial^{2} C}{\partial \bar{y}^{2}}\right)+\left(\frac{D_{T}}{T_{c}}\right)\left(\frac{\partial^{2} T_{f}}{\partial \bar{x}^{2}}+\frac{\partial^{2} T_{f}}{\partial \bar{y}^{2}}\right)$

9 where the above governing equations can be represent in the non-dimensional form by invoking the

1. following non-dimensional variables:

$$
x=\bar{x} / L, \quad y=\bar{y} / L, \quad \psi=\bar{\psi} / \alpha_{f}, \quad \phi=C / C_{0},
$$

$\theta_{f}=\left(T_{f}-T_{c}\right) /\left(T_{h}-T_{c}\right), \quad \theta_{p}=\left(T_{p}-T_{c}\right) /\left(T_{h}-T_{c}\right), \quad \theta_{s}=\left(T_{s}-T_{c}\right) /\left(T_{h}-T_{c}\right)$

ir as,

ir $\frac{\partial^{2} \psi}{\partial x^{2}}+\frac{\partial^{2} \psi}{\partial y^{2}}=-R a \frac{\partial \theta_{f}}{\partial x}+R a \cdot N r \frac{\partial \phi}{\partial x}$ 


$$
\begin{aligned}
& \frac{\partial \psi}{\partial y} \frac{\partial \theta_{f}}{\partial x}-\frac{\partial \psi}{\partial x} \frac{\partial \theta_{f}}{\partial y}=\varepsilon\left(\frac{\partial^{2} \theta_{f}}{\partial x^{2}}+\frac{\partial^{2} \theta_{f}}{\partial y^{2}}\right)+N b\left(\frac{\partial \phi}{\partial x} \frac{\partial \theta_{f}}{\partial x}+\frac{\partial \phi}{\partial y} \frac{\partial \theta_{f}}{\partial y}\right)+ \\
& N t\left[\left(\frac{\partial \theta_{f}}{\partial x}\right)^{2}+\left(\frac{\partial \theta_{f}}{\partial y}\right)^{2}\right]+N h p\left(\theta_{p}-\theta_{f}\right)+N h s\left(\theta_{s}-\theta_{f}\right)
\end{aligned}
$$

$\frac{\partial \psi}{\partial y} \frac{\partial \theta_{p}}{\partial x}-\frac{\partial \psi}{\partial x} \frac{\partial \theta_{p}}{\partial y}=\varepsilon_{p}\left(\frac{\partial^{2} \theta_{p}}{\partial x^{2}}+\frac{\partial^{2} \theta_{p}}{\partial y^{2}}\right)+N h p \cdot \gamma_{p}\left(\theta_{f}-\theta_{p}\right)$

$r \quad 0=\frac{\partial^{2} \theta_{s}}{\partial x^{2}}+\frac{\partial^{2} \theta_{s}}{\partial y^{2}}+N h s \cdot \gamma_{s}\left(\theta_{f}-\theta_{s}\right)$

$\varepsilon \quad \frac{\partial \psi}{\partial y} \frac{\partial \phi}{\partial x}-\frac{\partial \psi}{\partial x} \frac{\partial \phi}{\partial y}=\frac{1}{L e}\left(\frac{\partial^{2} \phi}{\partial x^{2}}+\frac{\partial^{2} \phi}{\partial y^{2}}\right)+\frac{N t}{L e \cdot N b}\left(\frac{\partial^{2} \theta_{f}}{\partial x^{2}}+\frac{\partial^{2} \theta_{f}}{\partial y^{2}}\right)$

- By using the non-dimensional parameters, the length and position of the heater and the aspect ratio of

1 the triangle can be represent in non-dimensional form as,

v $\quad Y_{p}=\frac{y_{p}}{H}, \quad H_{H}=\frac{h}{H}, \quad A R=\frac{L}{H}$

$\wedge \quad$ By considering the problem description and the schematic view of the model in Fig.1, the

9 boundary conditions for the problem in non-dimensional form are given by,

$\psi\left(-\frac{A R}{2}, 1-H_{H}\right)=0,\left.\quad \frac{\partial \theta_{f}}{\partial x}\right|_{\left(-\frac{A R}{2}, I-H_{H}\right)}=\left.\frac{\partial \theta_{p}}{\partial x}\right|_{\left(-\frac{A R}{2}, I-H_{H}\right)}=\left.\frac{\partial \theta_{s}}{\partial x}\right|_{\left(-\frac{A R}{2}, I-H_{H}\right)}=0,\left.\quad \frac{\partial \phi}{\partial x}\right|_{\left(-\frac{A R}{2}, I-H_{H}\right)}=0$

$\psi\left(-\frac{A R}{2}, H_{H}\right)=0, \theta_{f}\left(-\frac{A R}{2}, H_{H}\right)=\theta_{p}\left(-\frac{A R}{2}, H_{H}\right)=\theta_{s}\left(-\frac{A R}{2}, H_{H}\right)=1$,

ir $\left.\quad N b \frac{\partial \phi}{\partial x}\right|_{\left(-\frac{A R}{2}, H_{H}\right)}+\left.N t \frac{\partial \theta}{\partial x}\right|_{\left(-\frac{A R}{2}, H_{H}\right)}=0$

ir $\psi(x, 0)=0,\left.\frac{\partial \theta_{f}}{\partial y}\right|_{(x, 0)}=\left.\frac{\partial \theta_{p}}{\partial y}\right|_{(x, 0)}=\left.\frac{\partial \theta_{s}}{\partial y}\right|_{(x, 0)}=0,\left.\frac{\partial \phi}{\partial y}\right|_{(x, 0)}=0$ 


$$
\begin{aligned}
& \psi\left(x,-\frac{x}{A R}+\frac{1}{2}\right)=0, \theta_{f}\left(x,-\frac{x}{A R}+\frac{1}{2}\right)=\theta_{p}\left(x,-\frac{x}{A R}+\frac{1}{2}\right)=\theta_{s}\left(x,-\frac{x}{A R}+\frac{1}{2}\right)=0, \\
& \left.N b \frac{\partial \phi}{\partial n}\right|_{\left(x,-\frac{x}{A R}+\frac{1}{2}\right)}+\left.N t \frac{\partial \theta}{\partial n}\right|_{\left(x,-\frac{x}{A R}+\frac{1}{2}\right)}=0 \text { for }-A R / 2 \leq x \leq+A R / 2
\end{aligned}
$$

$r \quad$ where $\mathrm{Nb}, \mathrm{Nt}, \mathrm{Nr}$ and $\mathrm{Le}$ denote the Brownian motion parameter, thermophoresis parameter, buoyancy $\varepsilon \quad$ ratio parameter and Lewis number, respectively. The Nhp and Nhs show the interface heat transfer - parameters for the nanoparticles-base fluid and the porous matrix-base fluid, known as Nield numbers 1 [34]. Finally, $\varepsilon_{p}, \gamma_{p}$ and $\gamma_{s}$, depict a modified thermal diffusivity ratio for nanoparticles, modified thermal capacity ratio for nanoparticles and modified thermal conductivity ratio for porous phase. These parameters are defined as,

$$
\begin{aligned}
& N r=\frac{\left(\rho_{p}-\rho_{f 0}\right) C_{0}}{\rho_{f 0} \beta \Delta T\left(1-C_{0}\right)}, \quad N b=\frac{\tau D_{B} C_{0} \varepsilon}{\alpha_{f}}, \quad N t=\frac{\tau D_{T} \varepsilon \Delta T}{\alpha_{f} T_{c}}, \quad N h p=\frac{h_{f p} L^{2}}{k_{f}\left(1-C_{0}\right)}, \\
& N h s=\frac{h_{f s} L^{2}}{k_{f}\left(1-C_{0}\right)}, \quad \varepsilon_{p}=\frac{\alpha_{p} \varepsilon}{\alpha_{f}}, \quad \gamma_{p}=\frac{\left(1-C_{0}\right)(\rho c)_{f}}{C_{0}(\rho c)_{p}}, \quad \gamma_{s}=\frac{k_{f}\left(1-C_{0}\right)}{k_{s}(1-\varepsilon)}, \quad L e=\frac{\alpha_{f}}{D_{B} \varepsilon}
\end{aligned}
$$

1. Here, the physical quantities of interest are the local and average heat and mass transfer from the left vertical wall. The local Nusselt numbers for the base fluid, nanoparticles and the solid matrix, i.e. $N u_{f}$, ir $N u_{p}, N u_{s}$, and the local Sherwood number $S h$ for nanoparticles are defined as,

ir $\quad N u_{f}=-\left(\frac{\partial \theta_{f}}{\partial x}\right)_{x=-\frac{A R}{2}}, \quad N u_{p}=-\left(\frac{\partial \theta_{p}}{\partial x}\right)_{x=-\frac{A R}{2}}, \quad N u_{s}=-\left(\frac{\partial \theta_{s}}{\partial x}\right)_{x=-\frac{A R}{2}}, \quad S h=-\left(\frac{\partial \phi}{\partial x}\right)_{x=-\frac{A R}{2}}$

I $\quad$ The average Nusselt and Sherwood numbers for the left wall are defined as,

$10 \quad \overline{N u_{f}}=\int_{0}^{1} N u_{f} d y, \quad \overline{N u_{p}}=\int_{0}^{1} N u_{p} d y, \quad \overline{N u_{s}}=\int_{0}^{1} N u_{s} d y, \quad \overline{S h}=\int_{0}^{1} S h d y$

17 It is worth mentioning that Sherwood number is a function of temperature gradient of the fluid phase due to the adopted boundary condition for impermeability of surface to the nanoparticles, i.e. Eq (23) as 
1 $\frac{\partial \phi}{\partial x}=-\frac{N t}{N b} \frac{\partial \theta_{f}}{\partial x}$. Thus, the analysis of the local and average Sherwood number is easily possible through

$r \quad$ analysis of Nusselt number for the fluid phase. Therefore, the local and average Sherwood number are

r written as $S h=\frac{N t}{N b} N u_{f}$ and $\overline{S h}=\frac{N t}{N b} \overline{N u_{f}}$, and hence, the results will be strictly reported for Nusselt

$\leqslant \quad$ number.

$\circ$

3. Numerical method and validation

$\checkmark \quad$ The set of partial differential equations, Eqs. (14)- (18), and the corresponding boundary conditions at $\wedge \quad$ the walls, i.e. Eq. (23), are solved by employing the finite element method $[35,36]$. In this regard, the 9 governing equations were formulated in the weak form $[35,36]$. In the finite element method, the 1. quadratic elements and the Lagrange shape function were utilized [35]. The governing equations for 11 momentum, thermal energy of phases and the conservation of nanoparticles were fully coupled using ir damped Newton method [36]. A parallel sparse direct solver [37] was employed to solve the corresponding algebraic equations. The computations were continued until the the residuals for the each of the residual equations become smaller than $10^{-6}$. The solution procedure, in the form of an in-house computational fluid dynamics (CFD) code, have been validated successfully against the works of Sun and Pop [20], Baytas and Pop [38], Chamkha and Ismael [7], Chamkha et al. [39] and Costa [40] for natural convection in porous media in enclosures. More details regarding to the utilized finite element solution procedure, can be found in excellent references by Gross and Reusken [41] and Wriggers [36].

Review of the previous studies indicates that the magnitude of Brownian motion $(\mathrm{Nb})$ and thermophoresis $(N t)$ parameters are very small in the order of $10^{-6}$ [42 and 43]. The buoyancy ratio parameter $(\mathrm{Nr})$ is higher than unity, and the Lewis number is very large of the order of $10^{3}$ and higher due to very low magnitude of Brownian defensive coefficient in nanofluids [44 and 45]. The interface 
heat transfer parameters for the nanoparticles-base fluid and porous matrix-base fluid, Nield numbers (Nhp and Nhs), are higher than unity [46]. The modified thermal capacity ratio for nanoparticles $\left(\gamma_{p}\right)$ and

the modified thermal conductivity ratio for porous phase $\left(\gamma_{s}\right)$ are in the order of 10 . Finally, the modified thermal diffusivity ratio $\left(\varepsilon_{p}\right)$ is order of one. The geometric parameters $Y_{p}$ and $H_{H}$ can be varied about the range of 0 to 1 , and the aspect ratio is considered in the range of 0.1 to 10 . Finally, the Darcy Rayleigh number is considered in the order of 100 . Here, according to the discussed range of non-dimensional parameters, in the present study the results are reported for $R a=100, N b=10^{-6}, N t=10^{-6}, L e=1000, N r=5.0$, Nhs =10.0, Nhp=10.0, $\gamma_{s}=10.0, \gamma_{p}=10.0 \varepsilon=0.5, \varepsilon_{p}=1.0, Y_{P}=0.7, H t=0.5$ and $A R=1.0$ and otherwise the value of parameter will be stated. Table 1 shows the average Nusselt numbers of various phases for the mentioned typical case for different grid sizes. The results are reported for two interface heat transfer parameters of low interface heat interaction of $N h p=N h s=10$ and high interface thermal interaction of $N h p=N h s=20$. As seen in Table 1 , the grid size of $100 \times 100$ provides adequate accuracy for most of engineering applications and graphical representation of the results. Hence, the results of figures are obtained with a mesh consist of $100 \times 100$ grid points.

\section{Table 1}

Grid independency test for $R a=100, N b=10^{-6}, N t=10^{-6}, L e=1000, N r=5.0, \gamma_{s}=10.0, \gamma_{p}=10.0 \varepsilon=0.5$, $\varepsilon_{p}=1.0, Y_{P}=0.5, H t=0.7$ and $A R=1.0$.

\begin{tabular}{|c|c|c|c|c|c|c|}
\hline \multirow{2}{*}{ Grid Size } & \multicolumn{3}{|c|}{$N h p=N h s=10$} & \multicolumn{3}{|c|}{$N h p=N h s=20$} \\
\hline & $N u_{f}$ & $N u_{s}$ & $N u_{p}$ & $N u_{f}$ & $N u_{s}$ & $N u_{p}$ \\
\hline $50 \times 50$ & 5.27 & 3.72 & 4.25 & 5.22 & 3.89 & 4.38 \\
\hline $100 \times 100$ & 5.34 & 3.73 & 4.28 & 5.30 & 3.90 & 4.41 \\
\hline $150 \times 150$ & 5.37 & 3.74 & 4.29 & 5.32 & 3.90 & 4.43 \\
\hline $200 \times 200$ & 5.37 & 3.74 & 4.30 & 5.32 & 3.91 & 4.43 \\
\hline
\end{tabular}


The results of present study are compared with the classical benchmark study of heat transfer of r a pure fluid in a differentially heated square cavity by neglecting the Brownian motion and r thermophoresis effects and assuming local thermal equilibrium between phases. The average Nusselt $\leqslant \quad$ number results are shown in Table 2. As seen, in this case there is a very good agreement with the - $\quad$ previous studies available in literature.

7

1. Sun and Pop [20] have studied the convective heat transfer of nanofluids in a triangular 11 enclosure filled with a nanofluid-saturated porous media. The researchers have neglected the Brownian ir motion and thermophoresis effects and utilized a single-phase model for nanofluids. They have also it assumed local thermal equilibrium among all three phases of the nanoparticles, the base fluid and the Is porous matrix. Hence, by neglecting the Brownian motion and thermophoresis effects $(N b=N t=0)$, and 10 also by neglecting the temperature difference between phases, the present study reduces to the study of 
Sun and Pop [20]. In this case, a comparison between the results of present study and those reported by Sun and Pop [20] is performed in Fig. 2 for $A R=1$ and $\mathrm{H}_{\mathrm{H}}=0.4$ as well as $\mathrm{H}_{\mathrm{H}}=0.8$. In the Fig. 2 the results are reported for different values of Rayleigh number. This figure shows excellent agreement with the results available in literature.

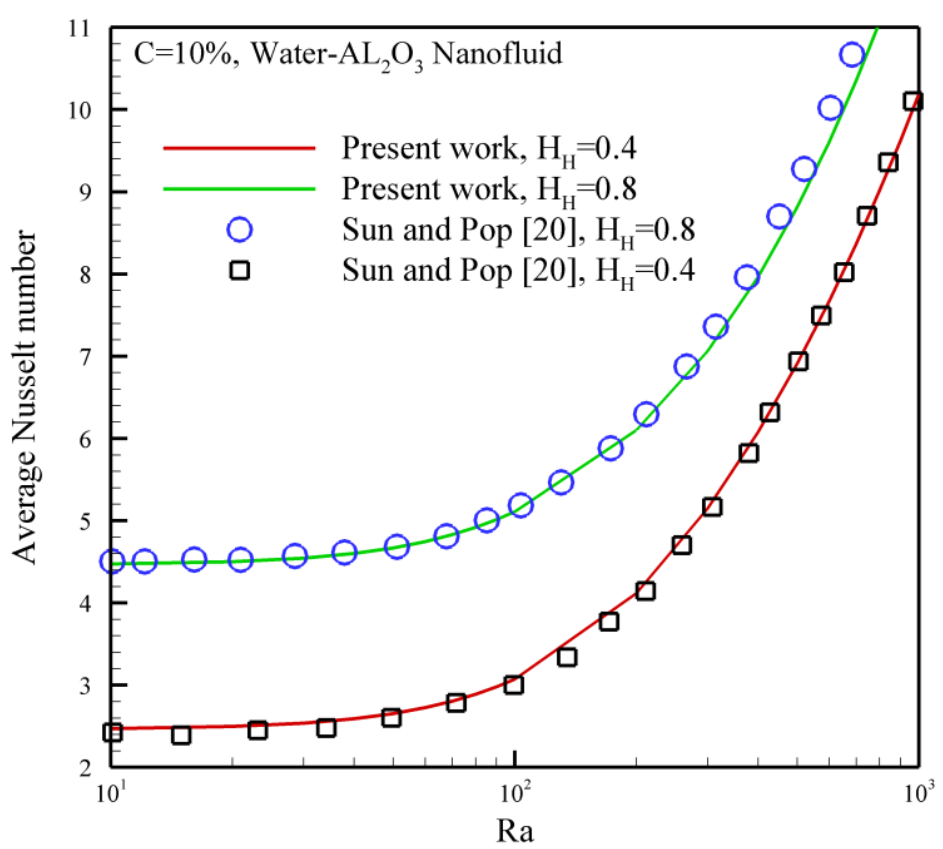

Fig. 2: Comparison of the evaluated average Nusselt number with the results of Sun and Pop [20].

Shermet and Pop [27] have studied the free convective heat transfer of nanofluids in a triangular cavity filled with porous media when the total of the vertical wall is at constant temperature of $T_{h}$. The authors have adopted the constant concentration of $C_{c}$ and $C_{h}$ for nanoparticles at the cold and hot walls ir and assumed local thermal equilibrium between phases. By considering the form of the obtained governing equations and boundary conditions in the study of Shermet and Pop [27], the results of

I present study are compared with those of the previous research in Fig. 3 for local Nusselt number at the 
vertical wall. Fig. 3 shows a good agreement between the present results and those reported by Shermet and Pop [27].

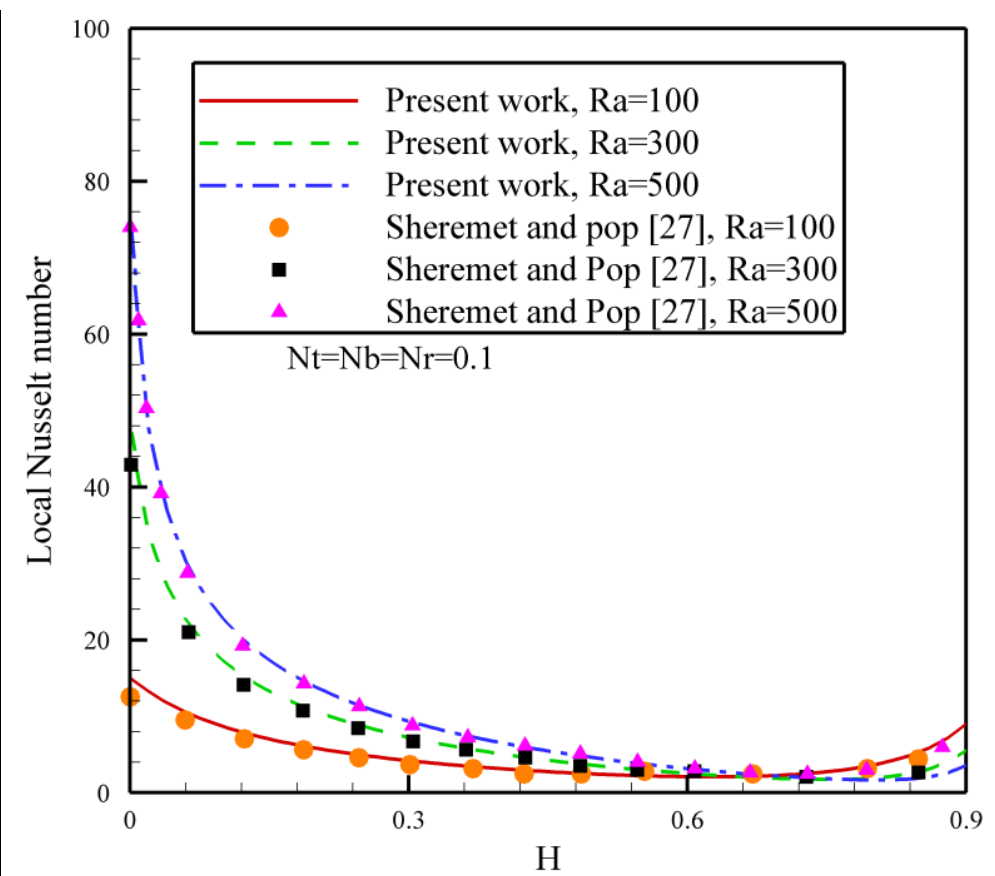

Fig. 3: A comparison between local Nusselt number of present work and Sheremet and Pop [27] when

$$
N r=N b=N t=0.1 \text {. }
$$

\section{Results and discussions}

Fig. 4 shows the effect of buoyancy ratio parameter $(\mathrm{Nr})$ on the streamlines in a triangular cavity. In this

1. figure, the streamlines are plotted for two values of $N r=0$ and $N r=10$. This figure clearly shows the clockwise circulation of the nanofluid inside the enclosure. The nanofluid next to the hot wall absorbs

ir the thermal energy and gets hot. The hot nanofluid is lighter than the cold one and as a result it moves in

ir upward direction. Then the hot flow reaches to the cold inclined wall, in which the nanofluid lose a part Is of its thermal energy and flows in downward direction. 
r.

r

r

r

Fig. 4 shows that the presence of the buoyancy effects due to mass transfer of nanoparticles induces a significant effect on the streamlines. Indeed, the thermophoresis force tends to move the nanoparticles away from the hot heater and push them into the cold inclined wall. It is clear that the migration of the heavy nanoparticles from a region to another region results in the buoyancy forces that consequently affect the streamlines.

Fig. 5 compares the non-dimensional temperature distribution of the three phases of nanoparticles $\left(\theta_{p}\right)$, the base fluid $\left(\theta_{f}\right)$ and the solid porous matrix $\left(\theta_{s}\right)$ in the enclosure. This figure indicates that near the top corner of the cavity, the temperature differences among the phases is small. In this region, the temperature profiles are very close together, and hence, the corresponding temperature gradients are strong. In contrast, at the right and the bottom areas of the triangular enclosure, the levels of temperature profiles are not very close together, and hence, the temperature gradients are also low. Fig. 5 illustrates the temperature profiles for each phase. The differences between the temperatures of the different phases is more obvious in the right and bottom of the enclosure where the flow velocities are small. The temperature distribution of the base fluid next to the heater is under the significant effect of fluid flow and shows a boundary layer shape. However, the temperature distribution in the solid matrix shows a distribution almost similar to the pure conduction in solids, but a variation due to the effect of the thermal interaction between the base fluid and nanoparticles is also obvious in the temperature distribution of this phase. The temperature distribution of nanoparticles almost follows the temperature distribution of the base fluid, but they are not identical as the interaction parameter (Nhp) between nanoparticles and base fluid phase is assumed finite.

Fig. 6 shows the concentration distribution of nanoparticles in the triangular cavity. As seen, the concentration of nanoparticles in the vicinity of the heater is low, and in contrast, it is high in the vicinity of the inclined cold wall. This distribution of concentration of nanoparticles is due to the 
1 thermophoresis forces, which tends to move the nanoparticles from the hot wall toward the cold one. In

r addition, Fig. 6 clearly shows that the concentration gradients of nanoparticles near the walls is high, but

$r \quad$ the concentration of nanoparticles in the core region of the enclosure is almost uniform. The high

$\varepsilon \quad$ concentration gradients next to the hot and cold walls is because of the high values of Lewis number.

- Indeed, the Brownian coefficient for diffusion of nanoparticles is very low, and hence, the concentration

1 gradient of nanoparticles is very high.

V

$\wedge$

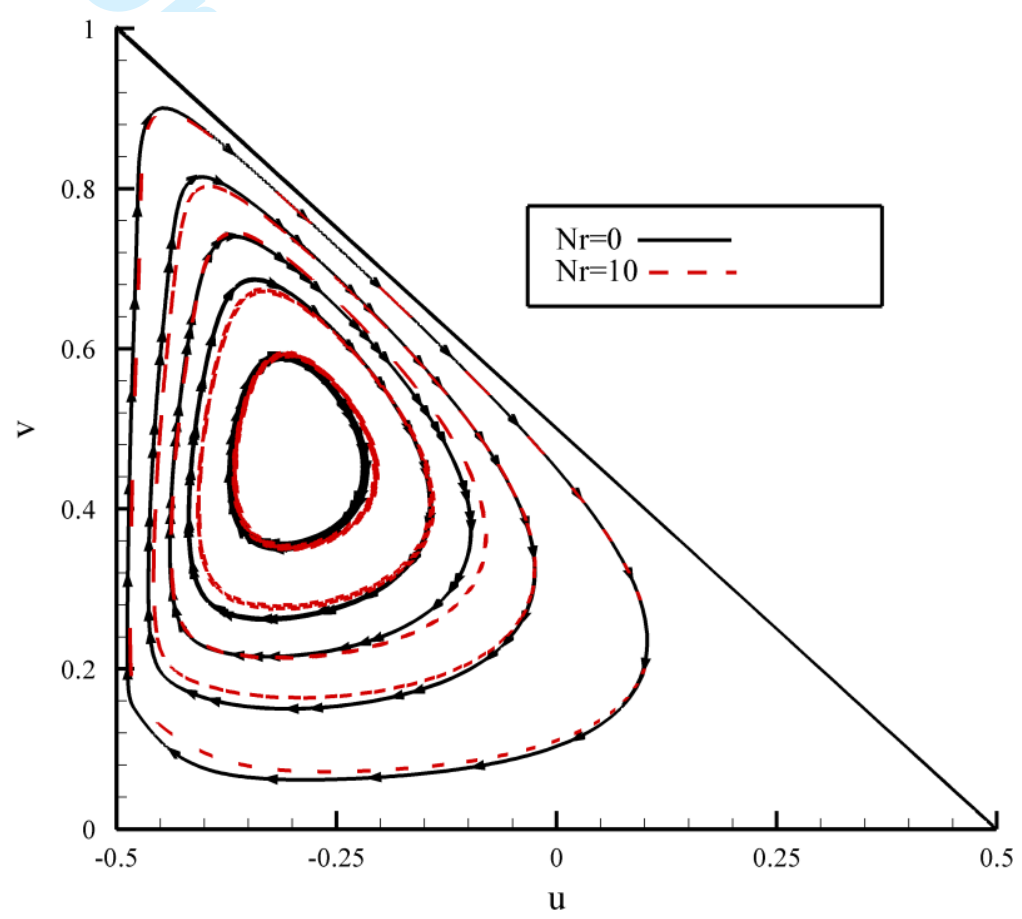

9

1. Fig. 4: A comparison between streamlines of nanofluid in $N r=0$ (black line) and $N r=10$ (red line). 


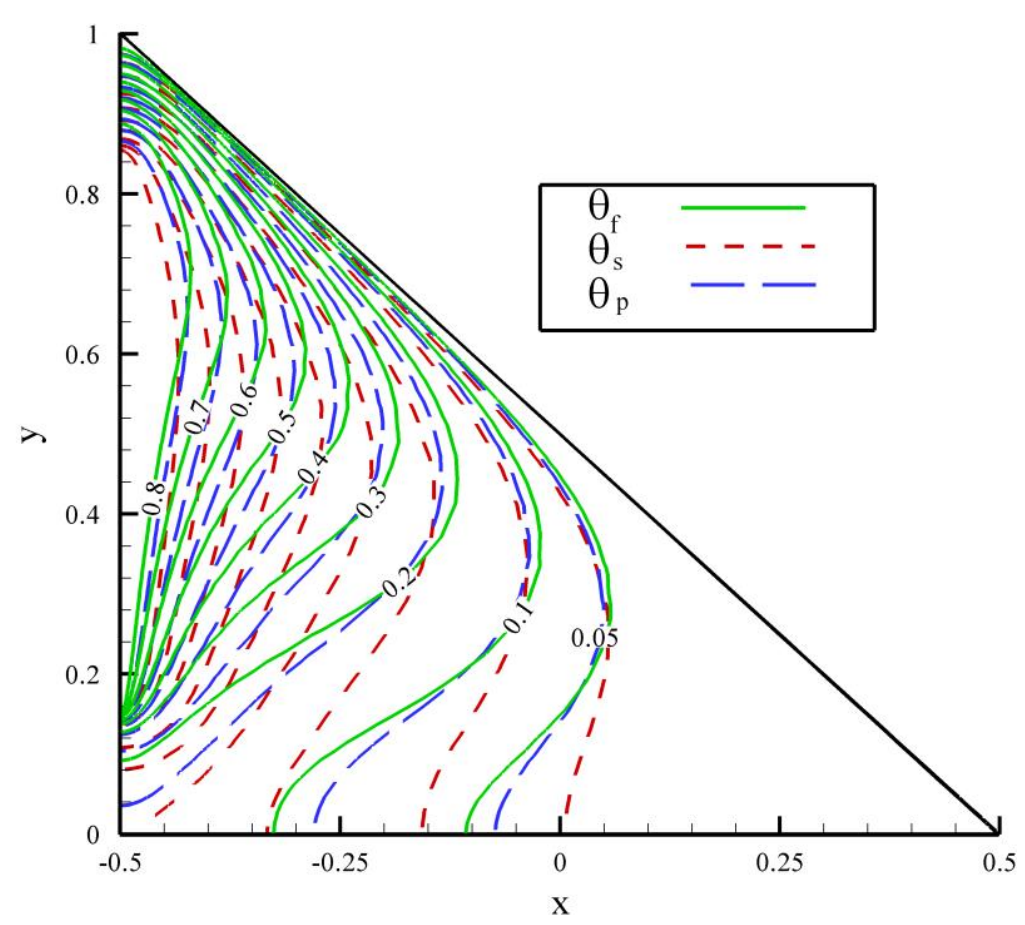

Fig.5: A comparison among isothermal contours of fluid (green line, $\theta_{f}$ ), solid (red dashed line, $\theta_{s}$ ), nanoparticles (blue long dashed, $\theta_{p}$ ).

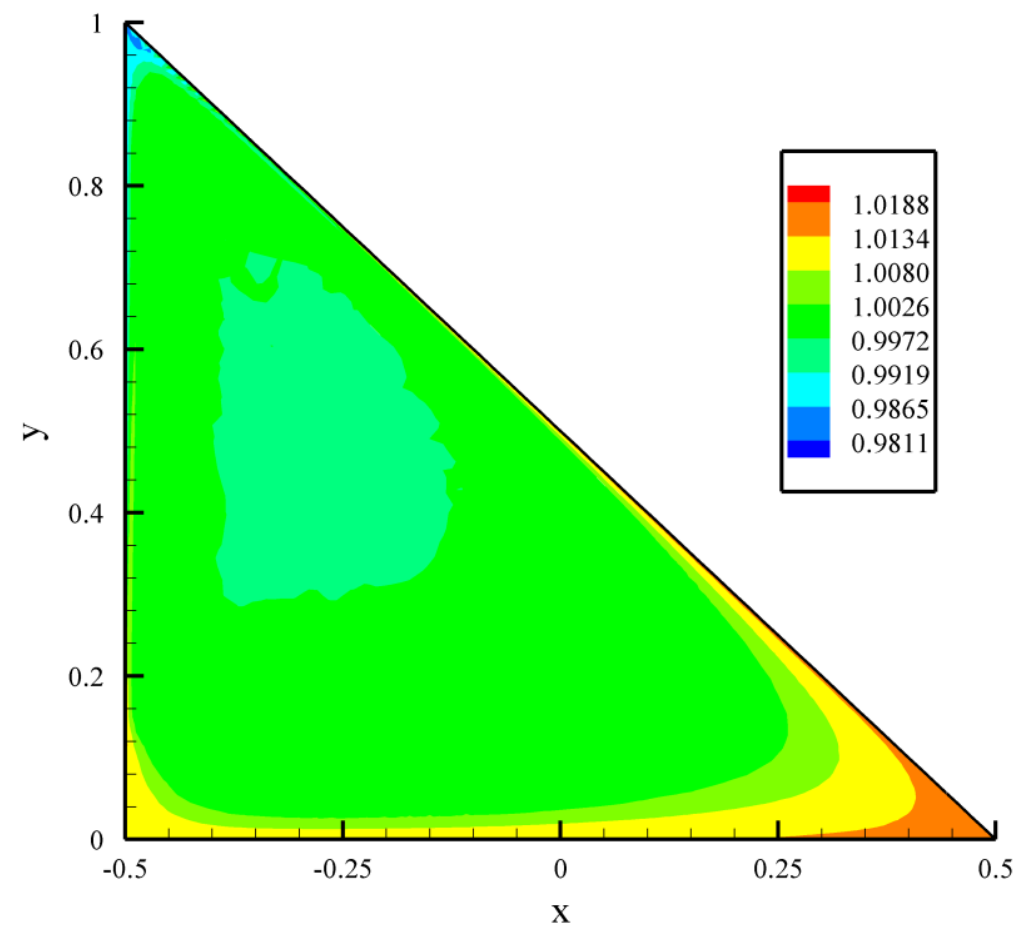

Fig.6: Contours of the concentration of nanoparticles in triangular cavity. 
1. the nanoparticles plays a significant role.

11

Figs. 7 shows the effect of interface heat parameters for nanoparticles, i.e. Nhp, on the temperature distribution in the nanoparticles phase. The isotherms for the base fluid when Nhp=10 (the typical case) are also plotted in this figure for the sake of comparison. Fig. 7 shows that the increase of the heat transfer parameter for nanoparticles tends to shift the nanoparticles phase isotherms toward the cold wall. Indeed, the increase of the interface parameter, i.e. Nhp, indicates a higher thermal interaction between the nanoparticles and the base fluid phases. By the increase of the interaction between nanoparticles and the base fluid, the nanoparticles tends to follow the temperature profiles of the base fluid phase. It is also interesting that the most dominant effect of the variation of Nhp is occurred next to the bottom adiabatic wall. This is where the flow is slow and the interaction between the fluid phase and Fig. 8 shows the effect of the interface heat parameter for the porous phase, i.e. Nhs, on the temperature distribution in the porous matrix phase. In this figure, the isotherms of the fluid phase for the default set of non-dimensional parameters are also plotted for the sake of comparison. This figure shows that the increase of the interface interaction between the base fluid phase and the porous medium phase tends to shift the isotherms into the base fluid temperature. 


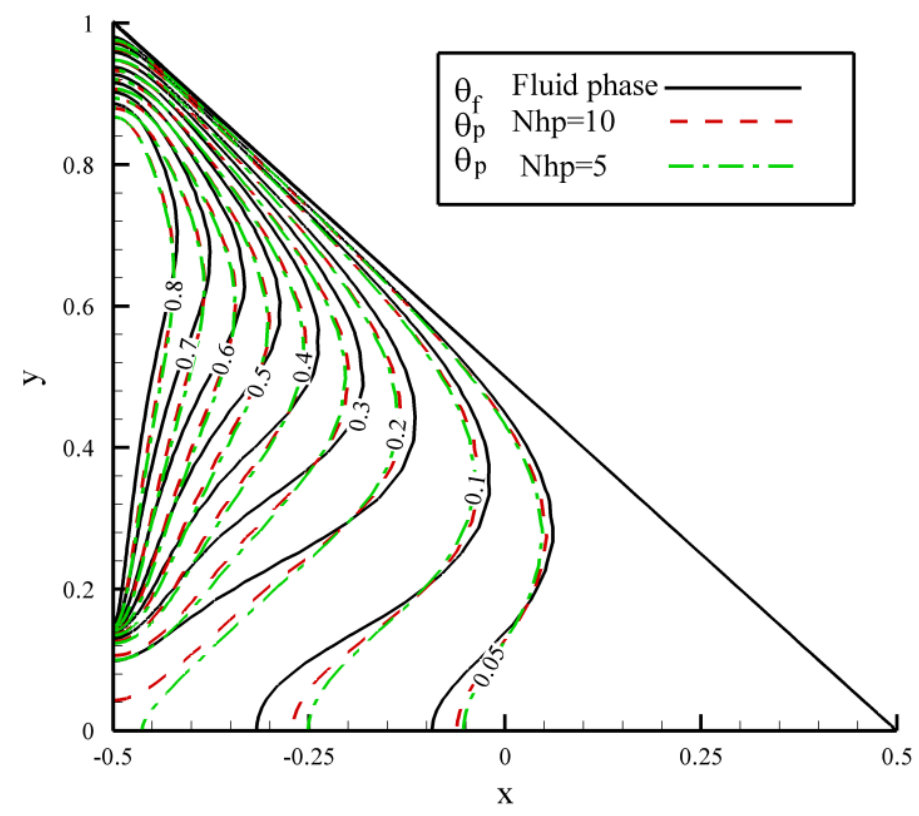

Fig.7: Evaluation of isothermal contours of nanoparticles in $N h p=5$ (red dashed line), Nhp=10 (black line).

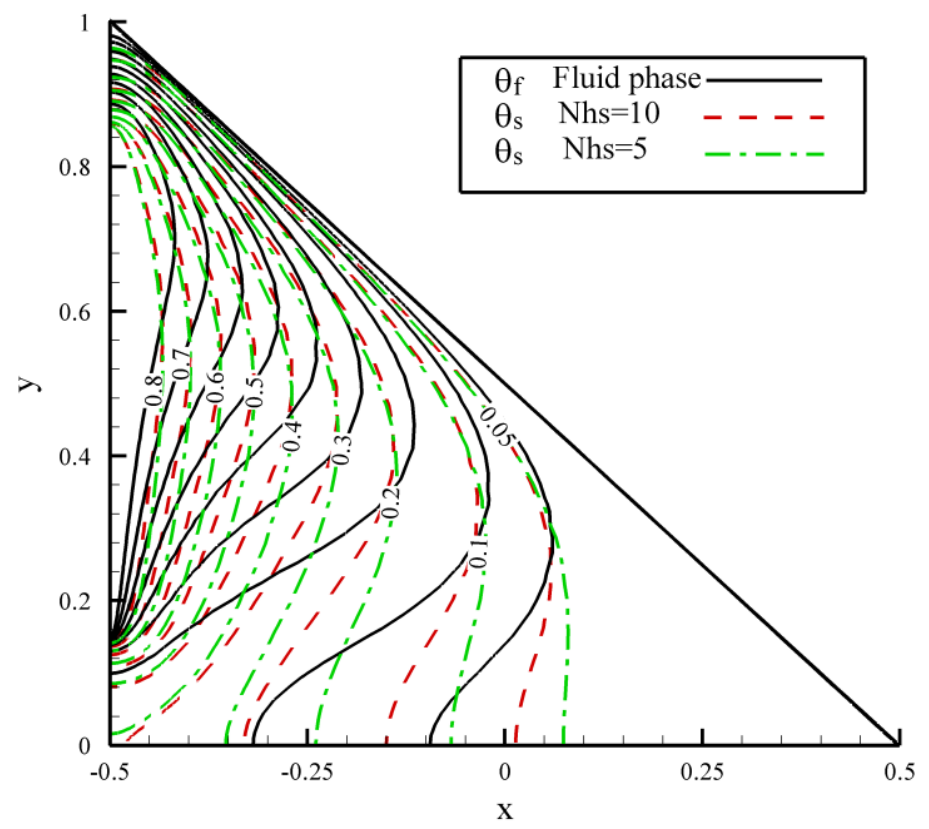

1

Fig. 8: Evaluation of isothermal contours of solid (porous media) for Nhs=5 (red dashed line) and Nhs $=10$ (black solid line). 
r.

The most significant temperature differences between the base fluid phase and the porous medium phase are in bottom of the enclosure where the nanofluid velocity is slow. The same trends of behaviors were also observed for the nanoparticles in Fig. 7, but here the difference between the isotherms is more distinct. This is because of the fact that the tiny nanoparticles would more easily follow the base fluid behavior rather than the solid porous matrix.

Fig. 9 compares the profiles of local Nusselt number of porous medium, base fluid and nanoparticles phases evaluated on the flush mounted heater at the vertical wall. The $\mathrm{x}$ axis in this figure is the arc length of the heater measured from the bottom of the heater. At the bottom of the heater where the fluid commenced to be heated, the local Nusselt number is significantly high. This is due to the fact that the fresh cooled nanofluid starts to absorb heat from the heater, and hence, the heat transfer rate due to diffusion mechanism is high. Then, the local Nusselt number falls rapidly to a fixed low level of average Nusselt number. This is where the temperature of the porous matrix, nanoparticles and the base fluid next to the wall starts raising. The raise of the temperature next to the wall reduces the temperature difference between wall and its surrounding, which results in the decrease of the heat transfer. In this region, the heat would be removed by both of the diffusive and advective mechanism, simultaneously. Finally, a sudden raise in local Nusselt number can be observed at the top of the heater. This region, is connected to the unheated surrounding areas, tending to strongly absorb heat from the heater element. This figure also shows that the local Nusselt number for the base fluid is very high at the bottom of the heater, but it is very low at the top part of the heater. Indeed, when the fresh and cold water reaches to the heater element, it tends to strongly absorb the heat from the heater element. In this case, the nanofluid not only carry the absorbed heat but also passes it into the surrounding media. In contrast, the nanofluid gets hot and hotter as it passes over the heater, and hence, the local Nusselt number reduces 
along the heater length moving from bottom to top. Next to the end of the heater, the liquid is under smooth effect of unheated surroundings media. However, it should be noted that the flow is flowing in upward direction. Thus, the effect of low temperature surrounding cannot be well passed through the fluid stream into the heater.

The porous matrix phase shows comparatively high values of local Nusselt number at the bottom and top parts of the heater. This is because of the fact that the heat transfer in porous matrix is dominant by the diffusion mechanism. Hence, at the top of the heater, the porous matrix can absorb the heat from the heater as well as the bottom of the heater. The smooth difference between the local Nusselt number for the porous phase at the bottom and the top of the heater is the result of the interaction between this 1. phase and the base fluid phase.

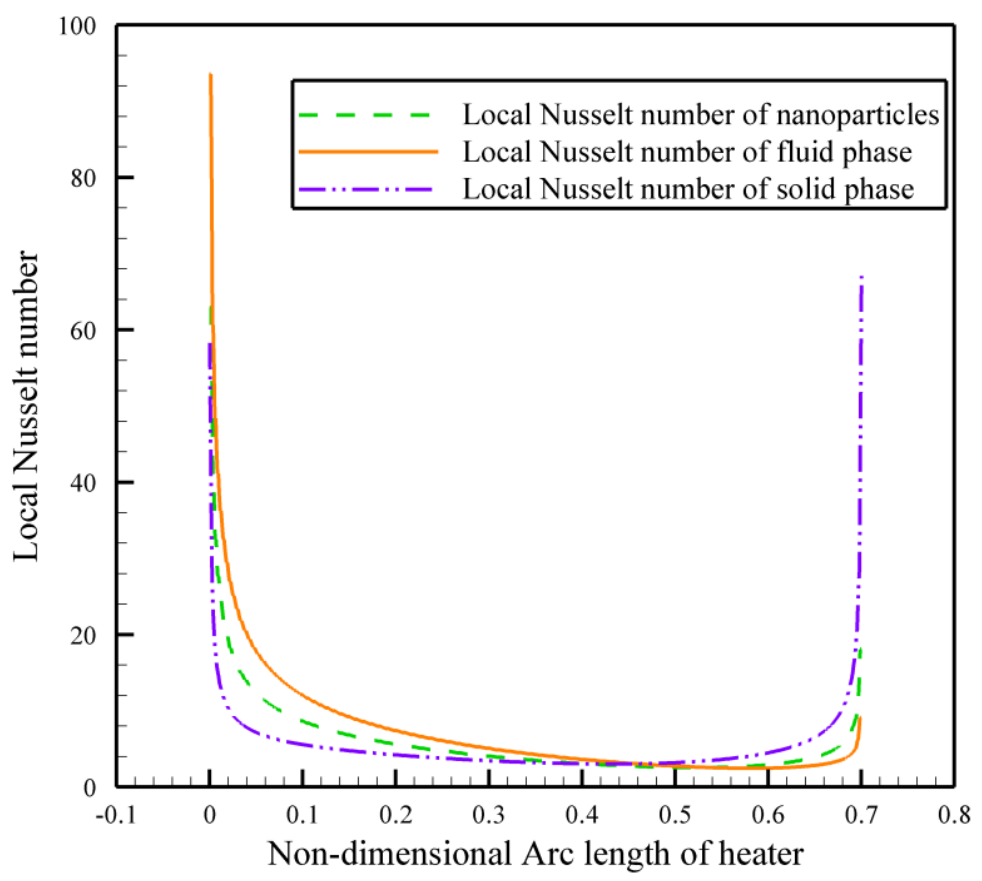

Fig. 9: Local Nusselt number of fluid phase $\left(N u_{f}\right)$, solid phase $\left(N u_{s}\right)$ and nanofluid $\left(N u_{p}\right)$ on heater in size of $H_{H}=0.7$. 
Fig. 10 compares the profiles of local Nusselt number for selected combinations of the interface $r \quad$ heat parameters. As seen, the increase of Nhp tends to smoothly shift the behavior of the local Nusselt $r$ profiles of nanoparticles phase towards the behavior of the base fluid phase (which was depicted in $\varepsilon \quad$ Fig.9). Similar to the nanoparticles phase, the increase of Nhs tends to shift the behaviour of the local - $\quad$ Nusselt number of the porous phase towards that of the base fluid phase, which was seen in Fig. 9.

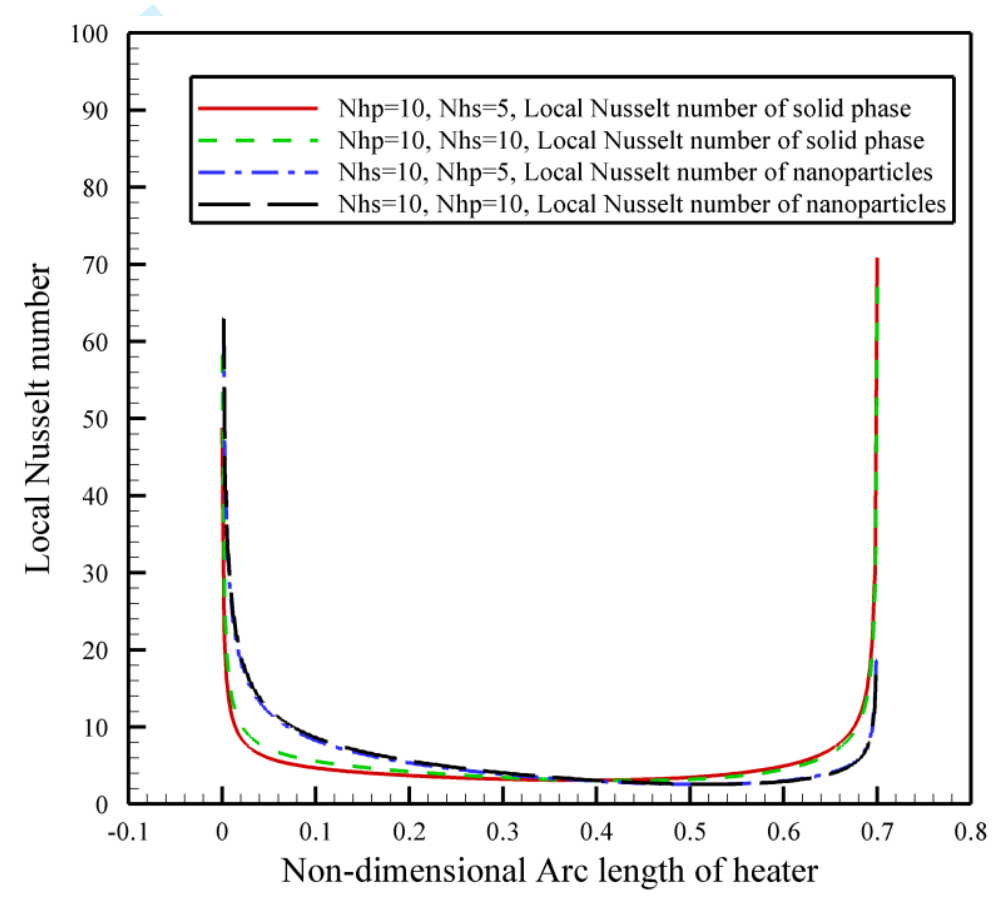

Fig. 10: Comparison between Local Nusselt number of solid phase and nanoparticles on heater in size of

$$
\mathrm{H}_{\mathrm{H}}=0.7 \text {. }
$$

Table 3 shows the effect of the enclosure size on the average Nusselt number of the studied phases. Figs. 11 and 12 show the corresponding streamlines and isotherms for a triangular enclosure of $A R=0.5$ and $A R=2.0$. It is worth mentioning that the corresponding streamlines and isotherms for $A R=1$

I $\quad$ were depicted in Figs. 4 and 5,previously. It is clear that the increase of the aspect ratio decreases the heat transfer in the enclosure. The increase of the aspect ratio results in a wider dead area in the bottom 
1 of the enclosure. Indeed, when the aspect ratio decreases, the hot and cold walls are get closer, and

$r$ hence, the Nusselt number increases.

$r \quad$ Table 3: Evaluation of average Nusslet number of fluid phase $\left(\overline{N u}_{s}\right)$, solid phase $\left(\overline{N u}_{p}\right)$ and nanofluid in several aspect ratio.

\begin{tabular}{cccc}
\hline $\mathbf{A R}$ & $\overline{N u}_{f}$ & $\overline{N u}_{s}$ & $\overline{N u}_{p}$ \\
& & & \\
\hline $\mathbf{0 . 5}$ & 5.72 & 4.46 & 5.19 \\
\hline $\mathbf{1}$ & 4.60 & 2.88 & 3.95 \\
\hline $\mathbf{2}$ & 3.94 & 2.23 & 3.38
\end{tabular}

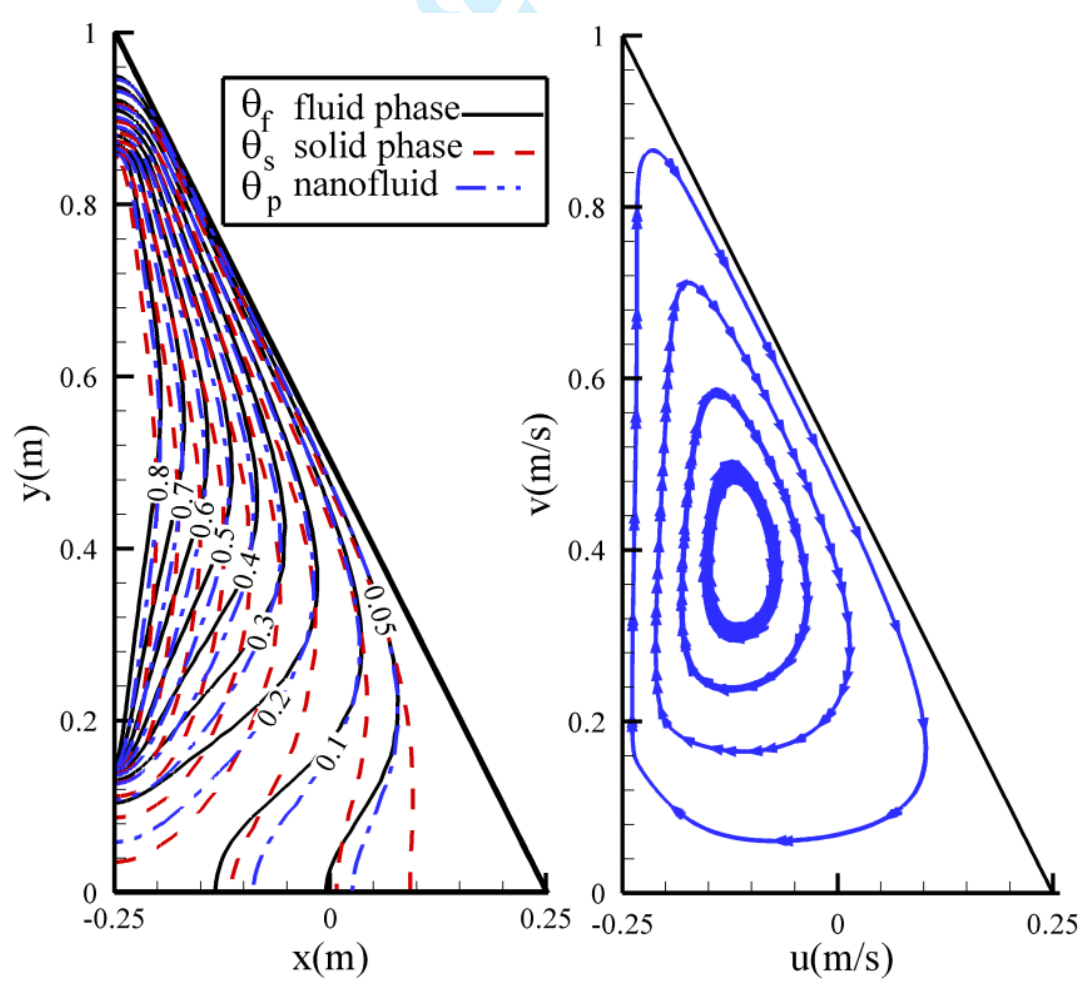

Fig. 11: Isotherm contours of fluid phase $\left(\theta_{f}\right)$, solid phase $\left(\theta_{s}\right)$ and nanoparticles $\left(\theta_{p}\right)$ (left side) and $\wedge \quad$ streamlines $(\psi)$ (right side) in $A R=0.5$. 
Figs. 13, 14 and 15 show the effect of interface parameters, i.e. Nhs and Nhp, on the average Nusselt r numbers of the fluid phase, the nanoparticles phase and the porous medium phase, respectively. Fig. 13 $\varepsilon \quad$ shows that the increase of the interface parameters, i.e. Nhs and $N h p$, reduces the heat transfer of the - fluid phase at the vertical wall (the raise of $\overline{N u}_{f}$ ). In fact, the increase of the interface heat transfer 1 parameters increases the interaction between the phases and reduces the temperature difference between $\checkmark \quad$ the wall and the fluid in the vicinity of the wall, which ultimately results in a lower rate of the heat $\wedge \quad$ transfer through the base fluid phase.
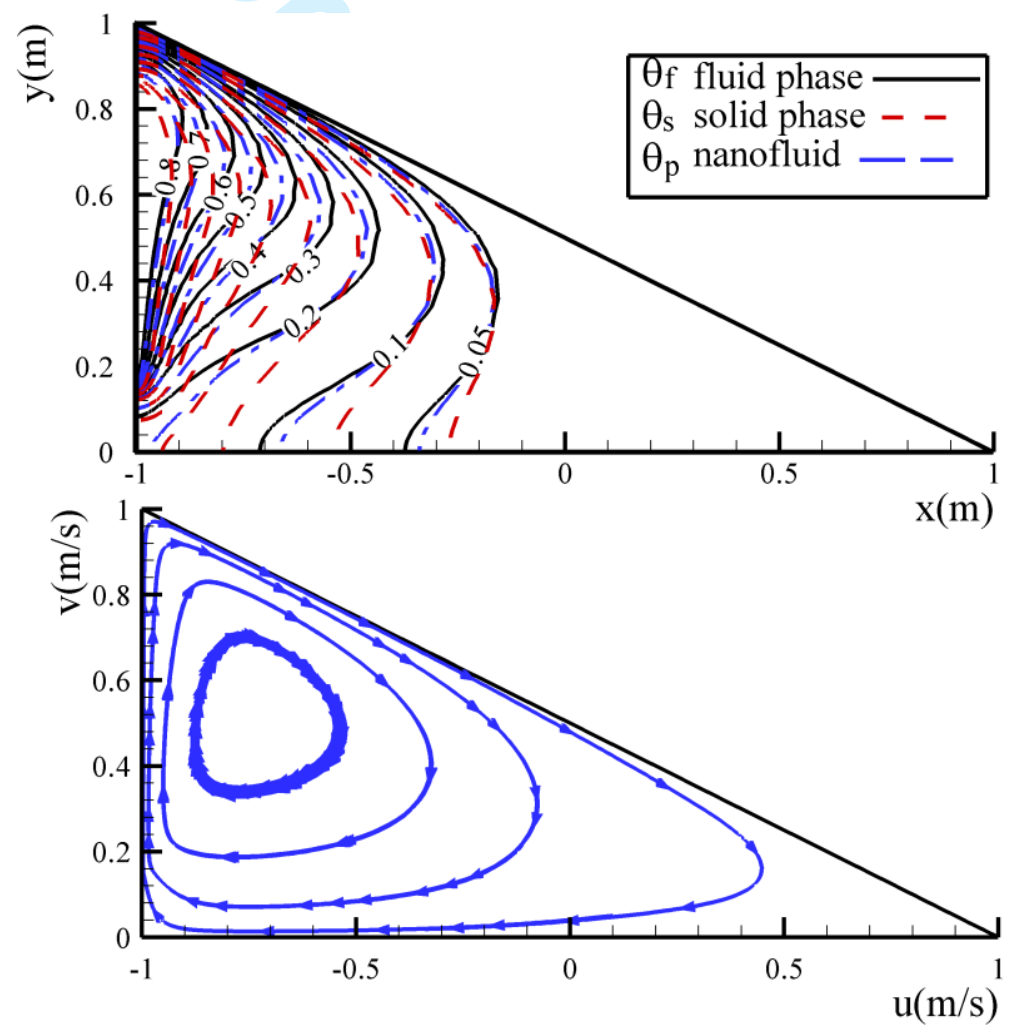

1. Fig. 12: Isotherm contours of fluid phase $\left(\theta_{f}\right)$, solid phase $\left(\theta_{s}\right)$ and nanoparticles $\left(\theta_{p}\right)$ (Top figure) and 11 streamlines $(\psi)$ (bottom figure) in $A R=2$. 
Fig. 14 reveals that the addition of the interface heat transfer parameter for nanoparticles, Nhp, would significantly raise the average Nusselt number for the nanoparticles phase. In fig. 13, it was found that $N u_{f}$ is a decreasing function of $N h p$, which shows that the interaction between the nanoparticles and fluid phase tends to reduce the temperature difference between the fluid phase and the hot wall. The decrease of the temperature difference between the hot wall and the base fluid phase means that the temperature of the base fluid next to the wall is increased by the interaction with nanoparticles. This is only possible when the temperature of the fluid phase be lower than the temperature of the nanoparticles, and in this case, the hot nanoparticles will lose a part of their thermal energy to the fluid phase. When the nanoparticles pass their thermal energy to their surrounding base fluid, their temperature falls down, and consequently, the temperature difference between nanoparticles and the hot wall increases. The increase of the temperature difference between the nanoparticles and the hot wall results in the increase of the heat transfer rate (the increase of $\overline{N u}_{p}$ ), and consequently, the gradient of the nanoparticles temperature phase raises. This outcome can be clearly seen Fig. 14.

Fig. 14 shows that the addition of the interface heat transfer parameter for the porous phase, Nhs, smoothly reduces the heat transfer rate in the nanoparticles phase $\left(\overline{N u}_{p}\right)$. The increase of interaction between the porous matrix and the fluid phase tends to increase the temperature of the fluid phase in the vicinity of the hot wall. When the temperature of the base fluid due to interaction with the porous matrix increases, the temperature of the fluid phase could be close to temperature of nanoparticles or higher; hence, the fluid phase would absorb a little amount of heat from the nanoparticles or passes some amount of heat to the nanoparticles. The results of Fig. 14 shows that for the high values of the interaction parameter between the base fluid and porous matrix phases, $N h s=10$, the fluid phase would pass some amount of thermal energy to the nanoparticles, and hence, the temperature of the 
1 nanoparticles is raised. The raise of the nanoparticles temperature next to the heater reduces the

r temperature gradient and consequently reduces the average Nusselt number for the nanoparticles phase.

Fig. 15 shows the effect of interface heat transfer parameters on the heat transfer rate of the $\varepsilon \quad$ porous medium phase. The augmentation of the interface heat transfer parameter, Nhs, for porous phase

- $\quad$ significantly increases the average Nusselt number for this phase. This is due the fact that the addition of

1 Nhs increases the thermal interaction between the fluid phase and the porous phase, which results in the

$\checkmark$ increase of the temperature of the fluid phase (the observed decrease of $\overline{N u}_{f}$ in Fig. 13) and

$\wedge$ simultaneously the reduction of the porous phase temperature. The reduction of the temperature of the

9 porous matrix, next to the heater, would consequently increase the average Nusselt number (temperature

1. gradient in porous phase). Finally, the increase of $N h p$ would smoothly decrease the average Nusselt

11 number of the porous phase. Indeed, the addition of $N h p$ increases the heat transfer interaction between

ir the base fluid and nanoparticles, which results in the increase of the fluid phase temperature. When the

ir temperature of the fluid phase raises, the absorbed heat from the porous matrix by base fluid reduces.

Is Hence, the temperature difference between the wall and the porous matrix remains low, and 10 consequently, the temperature gradient in the porous phase is low. 


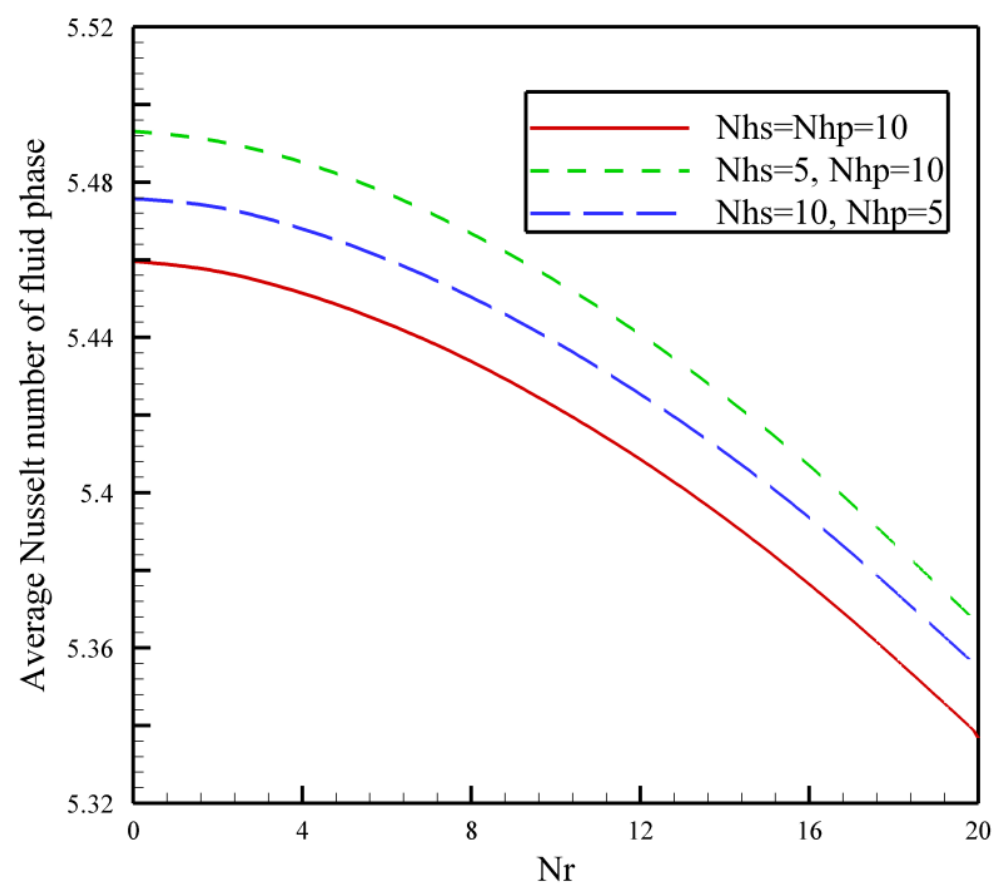

Fig.13: Average Nusselt number of fluid $\left(\overline{N u}_{f}\right)$ as a function buoyancy ratio $(N r)$.

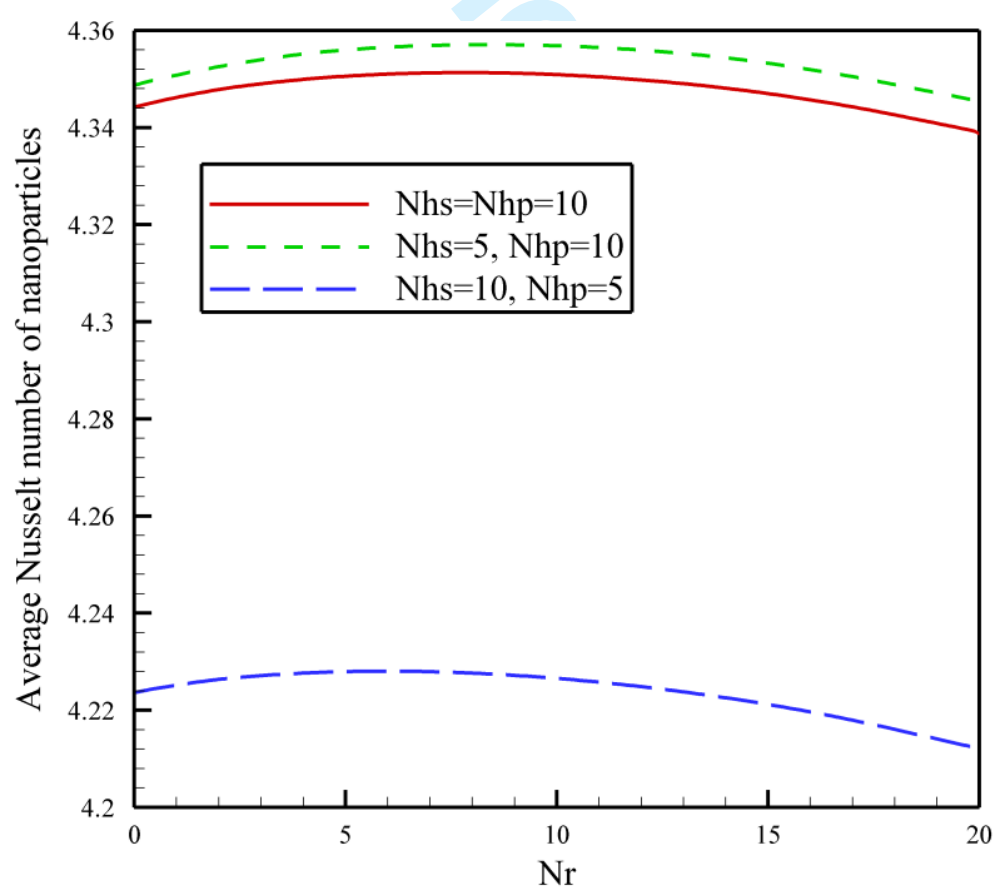

Fig.14: Average Nusselt number of nanoparticles $\left(\overline{N u}_{p}\right)$ as a function of buoyancy ratio $(N r)$. 


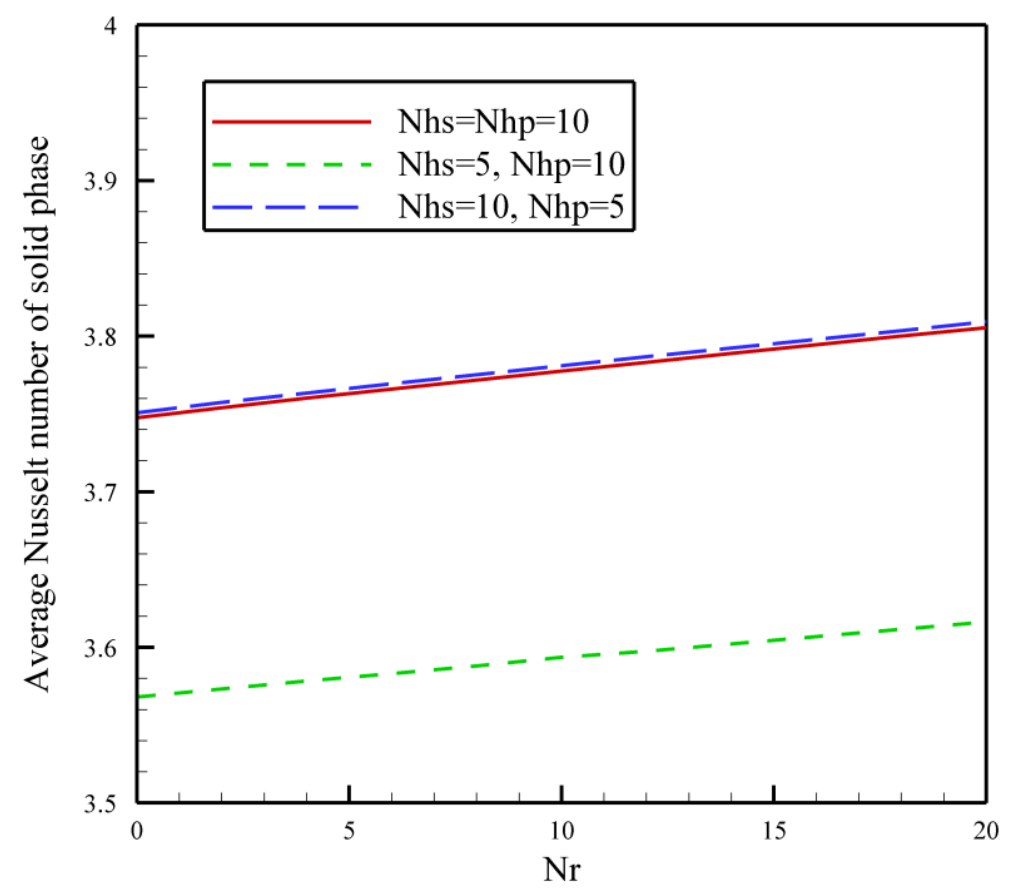

Fig.15: Average Nusselt number of porous media $\left(\overline{N u}_{s}\right)$ as a function of buoyancy ratio $(\mathrm{Nr})$.

Fig. 16 compares the magnitude of the average Nusselt numbers of different phases against various values of Rayleigh number. This figure interestingly depicts that the increase of Rayleigh number significantly boost the average Nusselt number of the base fluid phase and the nanoparticles phase. However, the average Nusselt number for the porous phase is a very smooth increasing function of Rayleigh number. The increase of Rayleigh number increases the fluid flow and thereby enhances the advective mechanisms, which results in the increase of the Nusselt number for the nanoparticles and the base fluid phases. However, the solid porous medium is stationery and does not directly incorporate in the advective mechanisms. The smooth variation of average Nusselt number of the porous phase is due to the interaction between the porous media and the fluid phase through the interface heat transfer mechanism for the porous phase (Nhs). 


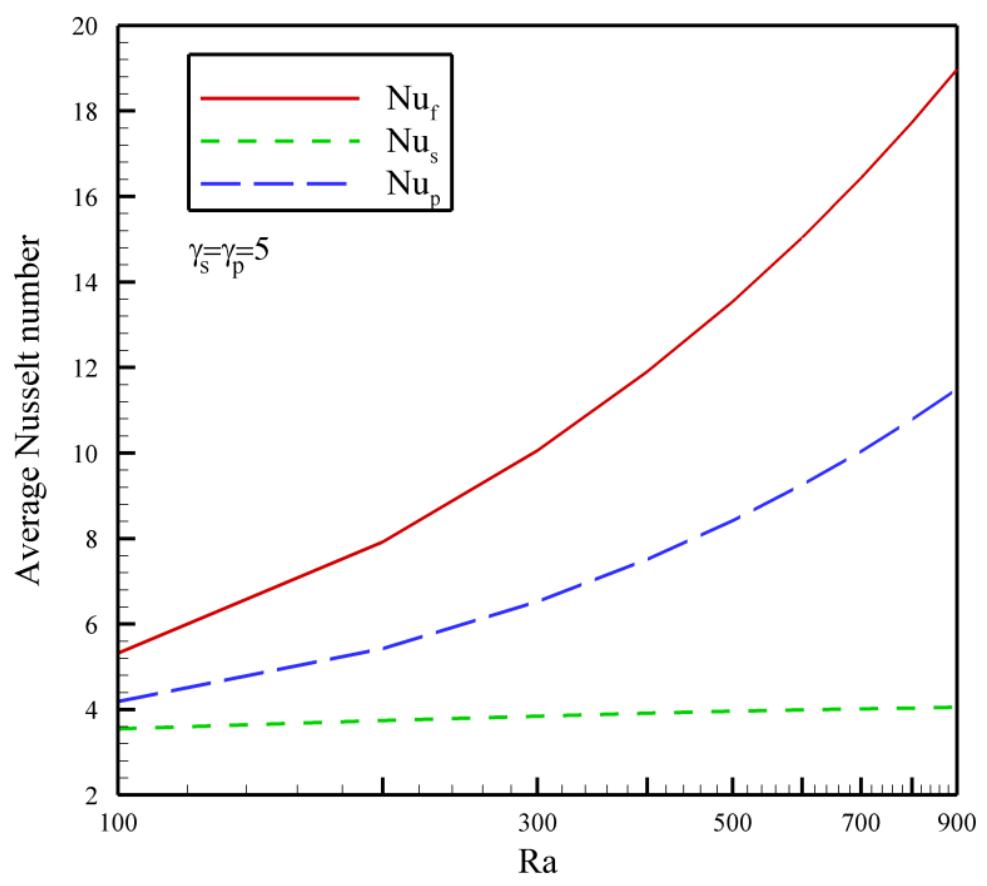

Fig.16: Average Nusselt number as a function of Rayleigh number.

Figs. 17, 18 and 19 show the effect of the modified heat capacity for nanoparticles $\left(\gamma_{p}\right)$ and the modified thermal conductivity for porous media $\left(\gamma_{s}\right)$ on the average Nusselt number of the three phases. These figures depict that the addition of $\gamma_{s}$ simultaneously increases all of the three the average Nusselt numbers. Indeed, the addition of $\gamma_{s}$ boosts the effect of the interaction of fluid and porous media at the porous medium side and tends to more effectively remove the heat from the wall and distribute it in the solid and nanoparticles phases.

Fig. 17 shows that the effect of variation of $\gamma_{p}$ on the average Nusselt number of the fluid phase depends on the magnitude of $\gamma_{s}$. When $\gamma_{s}$ is low, the raise of $\gamma_{p}$ decreases the average Nusselt number of the base fluid phase, but when $\gamma_{s}$ is high, the raise of $\gamma_{p}$ increases the average Nusselt number. Indeed, the increment of $\gamma_{p}$ tend to boost the effect of the interfacial interaction for the nanoparticles phase. When $\gamma_{s}$ is small (about unity), the temperature of the fluid phase is under the strong influence of the 
1 temperature distribution in the porous phase, which results in the decrease of average Nusselt number by

r the increase of $\gamma_{p}$.

Fig. 18 depicts that the addition of $\gamma_{p}$ shows an insignificant effect on the average Nusselt

$\leqslant$

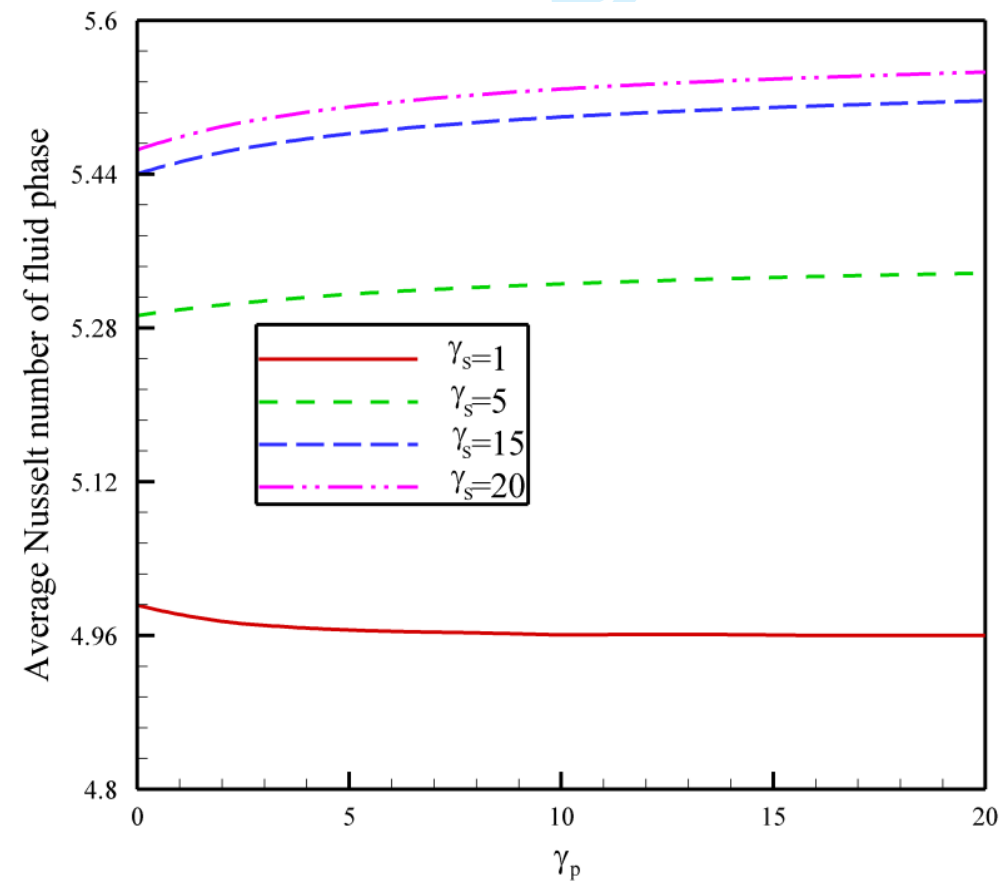


Fig.17: Average Nusselt number of fluid phase $\left(\overline{N u}_{f}\right)$ as a function of modified thermal capacity ratio of nanoparticles $\left(\gamma_{p}\right)$.

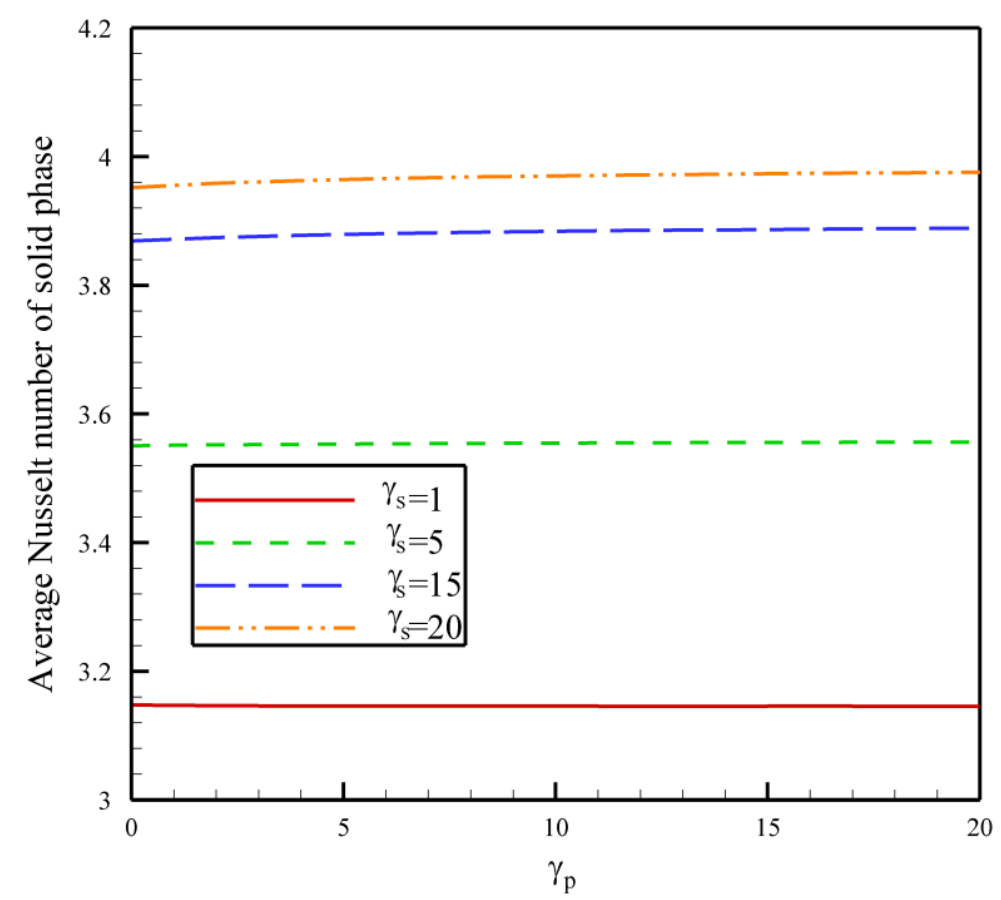

- Fig.18: average Nusselt number of solid phase $\left(\overline{N u}_{s}\right)$ as a function of modified thermal capacity ratio of nanoparticles $\left(\gamma_{p}\right)$. 


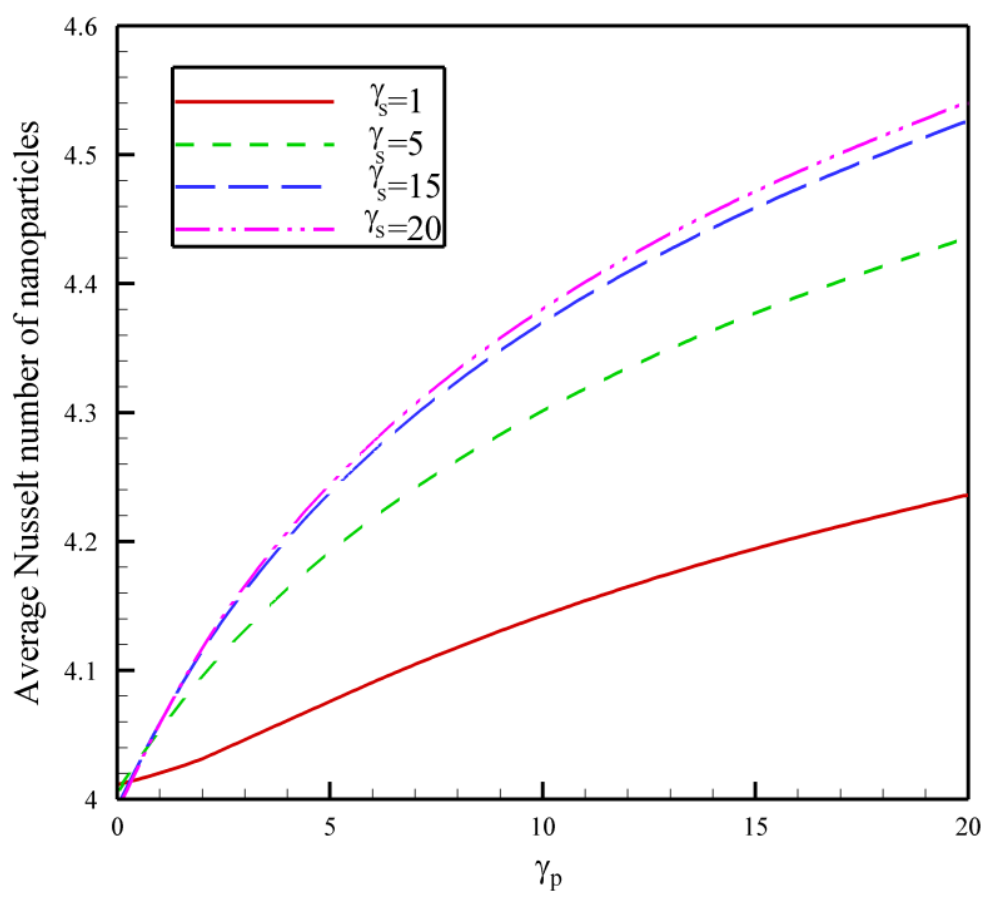

$\checkmark \quad$ Fig.19: average Nusselt number of nanoparticles $\left(\overline{N u}_{p}\right)$ as a function of modified thermal capacity ratio of nanoparticles $\left(\gamma_{p}\right)$.

Figs. 20, 21 and 22 depict the average Nusselt number of different phases as a function of $\gamma_{s}$ for various values of $\gamma_{p}$. These figures in agreement with the previous figures show that the increment of $\gamma_{s}$ is significant for all phases, but the increment of $\gamma_{p}$ is only significant for the nanoparticles phase. Fig. 20 shows a focal point (region) for average Nusselt number of the base fluid phase, which occurs for $\gamma_{s}$ about $\gamma_{s}=2.5$. This point could be of practical applications as the value of $\gamma_{s}=2.5$ is possible for nanofluids when the thermal conductivity of the porous matrix is low or the porosity of the porous medium is high. Fig. 22 also shows that the increase of $\gamma_{p}$ always increases the average Nusselt number for nanoparticles phase. Fig. 20 show that the increase of $\gamma_{p}$ (the modified heat capacity ratio for nanoparticles) starts to increase the average Nusselt number in the base fluid phase when the $\gamma_{s}$ is higher than unity. Thus, it could be concluded that for the $\gamma_{s}$ values higher than 2.5 the increase of $\gamma_{p}$ could boost the average Nusselt numbers for both phases of base fluid and nanoparticles. This is while the alteration of $\gamma_{p}$ does not show significant effect on the average Nusselt number of the porous phase. Fig. 
21 also indicates that the effect of $\gamma_{p}$ on the average Nusselt is very smooth due to the fact that alteration $r \quad$ of $\gamma_{p}$ indirectly affects the solid porous matrix through the alteration of the base fluid phase. This is

$r \quad$ while the increase of interaction between the base fluid and porous media $\left(\gamma_{s}\right)$ significantly increases the $\varepsilon \quad$ average Nusselt number of the porous phase.

○

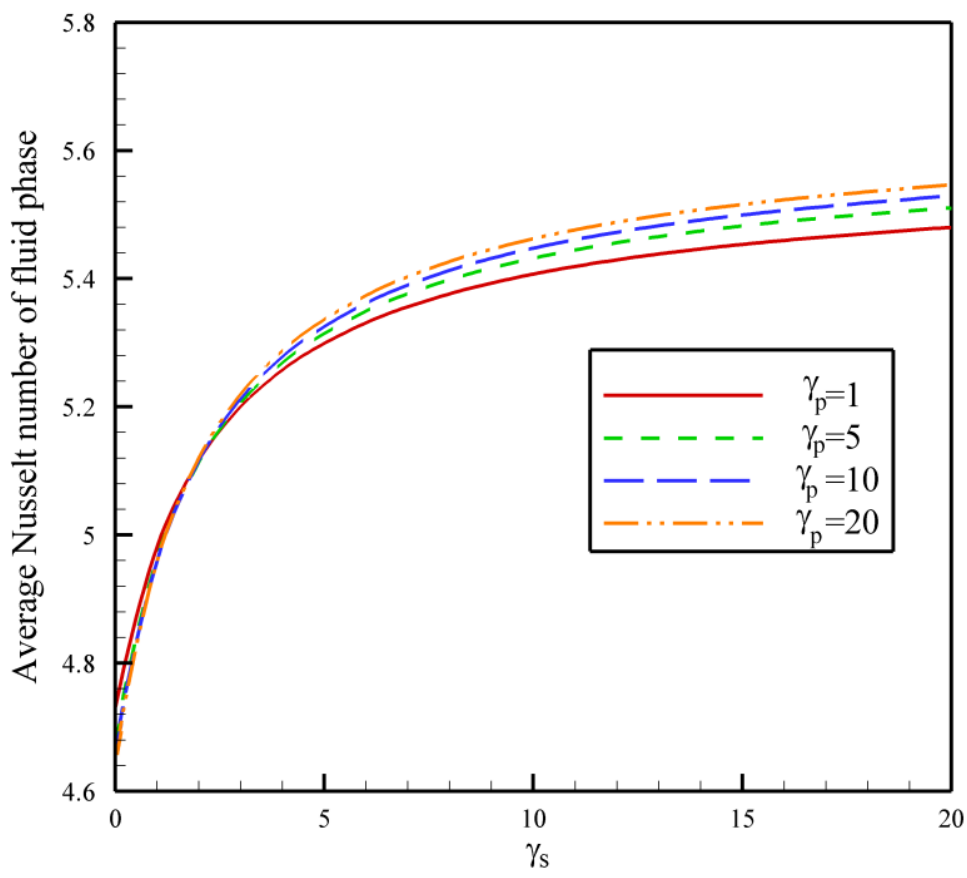

Fig.20: average Nusselt number of fluid phase $\left(\overline{N u}_{f}\right)$ in several modified thermal capacity ratio of nanoparticles $\left(\gamma_{\mathrm{p}}\right)$ as a function of modified thermal conductivity ratio of porous phase $\left(\gamma_{s}\right)$. 


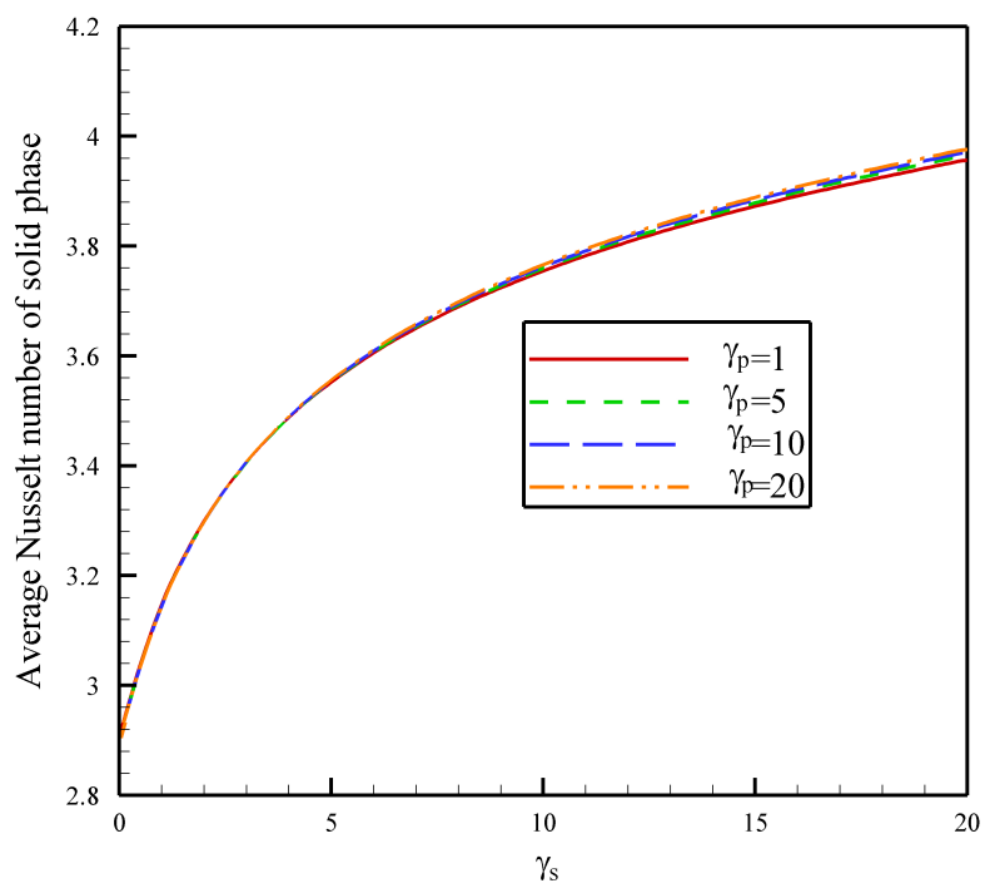

$\checkmark$ Fig.21: Average Nusselt number of solid porous media $\left(\overline{N u}_{s}\right)$ in several modified thermal capacity ratio of nanoparticles $\left(\gamma_{p}\right)$ as a function of modified thermal conductivity ratio of porous phase $\left(\gamma_{s}\right)$.

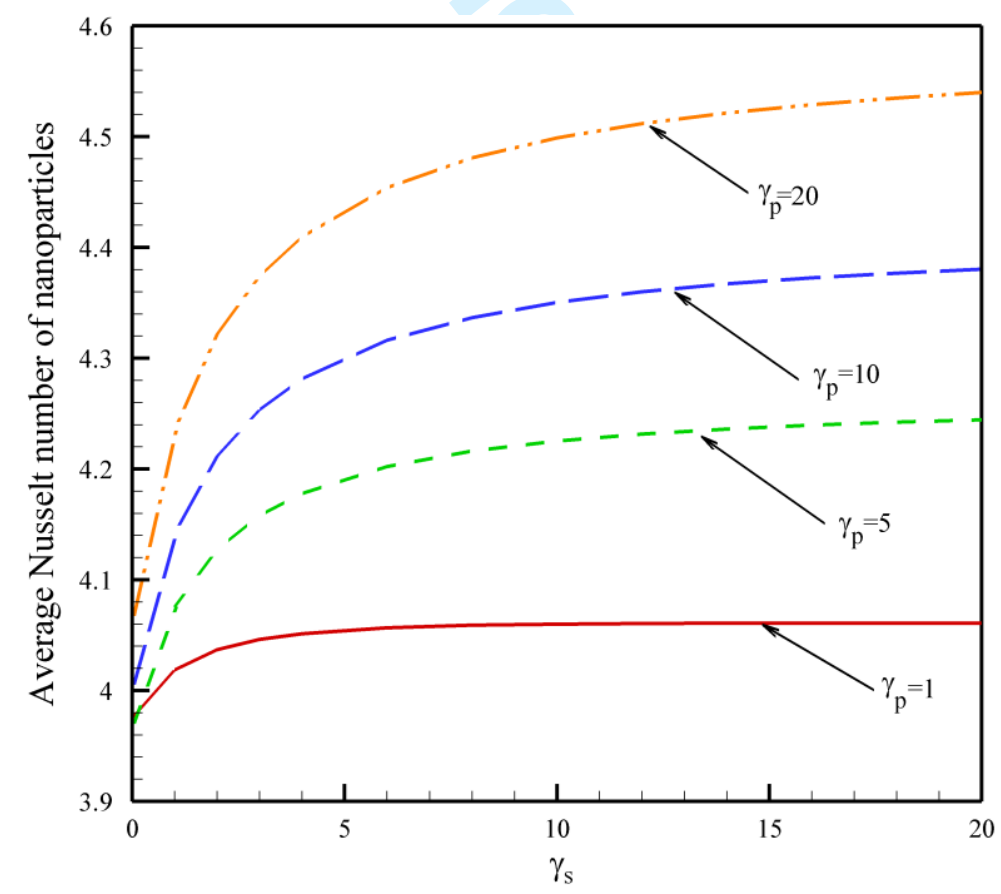

- Fig.22: average Nusselt number of nanoparticles $\left(\overline{N u}_{p}\right)$ in several modified thermal capacity ratio of nanoparticles $\left(\gamma_{p}\right)$ as a function of modified thermal conductivity ratio of porous media $\left(\gamma_{s}\right)$. 


\section{Conclusion}

$r$. 3 - The increase of the modified conductivity ratio parameter $\left(\gamma_{s}\right)$ would enhances the average Nusselt

The free convective heat and mass transfer of nanofluids in a triangular cavity filled with a saturated porous medium was theoretically analyzed. There was a flush heater mounted on a part of the vertical wall while the inclined wall was kept cold. A local thermal non-equilibrium model, incorporating the three-heat equations model, was employed for thermal energy of three phases of base fluid, nanoparticles and the porous medium. The drift flux of nanoparticles phase was modeled by using Buongiorno's nanofluid model, incorporating the Brownian motion and thermophoresis effects. The governing equations were transformed into the non-dimensional form, and they have been solved by using the finite element method. The main outcomes of the present study can be summarize as follows:

1- As the buoyancy ratio increases, the average Nusselt number for fluid phase decreases, for porous phase increases and for nanoparticles phase first increases and then decreases.

2- As the Rayleigh number increases, the average Nusselt number for base fluid rapidly increases, but the average Nusselt number of the porous phase is almost independent of the variation of Rayleigh number. The nanoparticles phase would follow the behavior of the base fluid phase but with a smoother slope.

number of all three phases, simultaneously. However, the raise of the heat capacity ratio $\left(\gamma_{p}\right)$ would solely induce a significant enhancement on the average Nusselt number of the nanoparticles phase. 
4- The augmentation of the heater size results in better heat transfer as it increases the average Nusselt number for all three phases simultaneously.

5- The increase of the aspect ratio of the enclosure reduces the average Nusselt number of all three phases. Hence, the enclosures with a low aspect ratio could result in a higher heat transfer rate.

\section{Acknowledgement}

The authors acknowledge the financial support of Dezful Branch, Islamic Azad University, Dezful, Iran and Iran Nanotechnology Initiative Council (INIC) for the support of the present study. The authors are tankful to Sheikh Bahaei National High Performance Computing Center (SBNHPCC) for providing computational resources, supported by scientific and technological department of presidential office and Isfahan University of Technology (IUT). Authors wish to appreciate the very component Reviewers for their careful revision of the present study.

\section{References}

[1] A.C. Baytas and I. Pop, (1999), Int. J. Heat Mass Transf. 42 (1999). doi. 10.1016_s0017-9310 (98) 00208-7.

[2] N. Saeid and I. Pop, J. Porous Media 8, 1 (2005). 10.1615/JPorMedia.v8.i1.50.

[3] T. Basak, S. Roy, T. Paul and I. Pop, (2006), Int. J. Heat Mass Transf. 49, 7 (2006). doi:10.1016/j.ijheatmasstransfer.2005.09.018

[4] M. Sathiyamoorthy, T. Basak, S. Roy and I. Pop, Int. J. Heat Mass Transf. 50, 9 (2007). doi:10.1016/j.ijheatmasstransfer.2006.10.010. 
[6] T. Basak, S. Roy and A.J. Chamkha, Int. Commun. Heat Mass Transf. 39 (2012). doi:10.1016/j.icheatmasstransfer.2012.03.022.

[7] A.J. Chamkha and M.A. Ismael, Numer. Heat Transf. Part A: Applications 63, 2 (2013). doi: $10.1080 / 10407782.2012 .724327$.

[8] D.B. Ingham and I. Pop, (Eds), Transport phenomena in porous media III, Elsevier, Oxford (2005).

[9] K. Vafai, (Ed.), Handbook of porous media, Crc Press, (2005).

[10] D.A. Nield and A. Bejan, Mechanics of Fluid Flow through a Porous Medium, Springer New York, pp. 1-29, (2013).

[11] G. Huminic and A. Huminic, Renew. Sustain Energy Rev. 16, 8 (2012). http://doi.org/10.1016/j.rser.2012.05.023.

[12] R. Saidur, K.Y. Leong and H.A. Mohammad, Renew. Sustain. Energy Rev. 15 (2011). doi:10.1016/j.rser.2010.11.035.

[13] A. Malvandi, D. Ganji and A. Malvandi. Int. J. Sediment Res. 29, 3 (2014). doi: 10.1016/S10016279(14)60056-1.

[14] A. Malvandi, J. Magn. Magn. Mater. 406 (2016). http://dx.doi.org/10.1016/j.jmmm.2016.01.008.

17 [15] I. Mustafa, T. Javed and A. Majeed, Can. J. Phys. (ja), (2015). doi: 10.1139/cjp-2014-0689.

iv

$1 \wedge$

19

$r$.

r)

rY

r

[16] M.F. Iqbal, K. Ali and M. Ashraf, Can. J. Phys. 93, 3. (2014). doi.10.1139/cjp-2014-0243.

[17] K. Kumar Ch, and S. Bandari, Can. J. Phys. 92, 12 (2014). doi.10.1139/cjp-2013-0508.

[18] M. M. Rashidi, N. Freidoonimehr, A. Hosseini, O. Anwar Bég, and T.K. Hung, Meccanica 49, 2 (2014). doi. 10.1007/s11012-013-98059.

[19] M.M. Rashidi, A. Hosseini, I. Pop, S. Kumar and N. Freidoonimehr, Appl. Math. Mech. 35, 7 (2014). doi. 10.1007/s10483-014-1839-9.

[20] Q. Sun and I. Pop, Int. J. Therm. Sci. 50 (2011). doi:10.1016/j.ijthermalsci.2011.06.005 
1 [21] M.A. Sheremet, T. Grosan and I. Pop, Transp. Porous Media 106, 3 (2015a). doi. 10/1007/s11242r $\quad 014-0145-3$.

r [22] M.A. Sheremet, S. Dinarvand and I. Pop, Physica E: Low-dimensional Systems and Nanostructures \& $\quad 69$ (2015b). http://dx.doi.org/10.1016/j.physe.2015.02.005.

- [23] M. Ghalambaz, M.A. Sheremet and I. Pop, (2015), PlOS ONE 10, 5 (2015). 7 doi:10.1371/journal.pone.0126486.

[24] M.A. Sheremet and I. Pop, Transp. Porous Media 105, 2 (2014a). doi. 10.1007/s11242-014-0375-7.

[25] J. Buongiorno, J. Heat Transf. 128, 3 (2006). doi:10.1115/1.2150834.

[26] M.A. Sheremet, T. Groşan and I. Pop, J. Heat Transf. 136, 8 (2014). doi: 10.1115/1.4027355.

[27] M.A. Sheremet and I. Pop, Int. J. Num. Methods Heat Fluid Flow 25, 5 (2014b). http://dx.doi.org/10.1108/HFF-06-2014-0181.

[28] A.V. Kuznetsov and D.A. Nield, Transp. Porous Media 83, 2 (2010). doi. 10.1007/s11242-0099452-8.

[29] A.V. Kuznetsov and D.A. Nield, Int. J. Heat Mass Transf. 65 (2013). doi. 10.1016/j.ijheatmasstransfer.2013.06.054.

[30] D.A. Nield and A.V. Kuznetsov, Int. J. Heat Mass Transf. 52, 25 (2009). doi. 10.1016/j.ijheatmasstransfer.2009.07.024.

[31] D.A. Nield and A.V. Kuznetsov, Int. J. Heat Mass Transf. 68 (2014). doi. 10.1016/j.ijheat mass transfer.2013.09.026.

[32] D.Y. Tzou, J. Heat Transfer 130, 7 (2008a). doi:10.1115/1.2908427.

[33] D.Y. Tzou, Int. J. Heat Mass Transf. 51, 11 (2008b). doi:10.1016/j.ijheatmasstransfer.2007.09.014. 
[34] P. Vadász, (Ed.), Emerging Topics in Heat and Mass Transfer in Porous Media: From Bioengineering and Microelectronics to Nanotechnology Springer Science \& Business Media Vol. 22 (2008).

[35] S. Rao, The finite element method in engineering, Butterworth-Heinemann, (2005).

[36] P. Wriggers, Nonlinear finite element methods, Springer Science \& Business Media (2008).

[37] P.R. Amestoy, I.S. Duff and J.Y. L'Excellent, Comput. methods appl. mech. eng. 184, 2 (2000). doi: 10.1016/S0045-7825(99)00242-X

[38] A.C. Baytas and I. Pop, Int. J. Therm. Sci. 41, 9 (2002). doi: 10.1016/S1290-0729(02)01379-0.

[39] A.J. Chamkha, M.A. Mansour and S.E. Ahmed, Heat Mass Transf. 46, 7 (2010). doi: 10.1007/s00231-010-0622-6.

[40] V.A.F. Costa, Int. J. Heat Mass Transf. 47, 12 (2004). doi: 10.1016/j.ijheatmasstransfer.2003.11.031.

[41] S. Gross and A. Reusken, Numerical methods for two-phase incompressible flows, Springer Science \& Business Media 40 (2011).

[42] H. Zargartalebi, A. Noghrehabadi, M. Ghalambaz and I. Pop, Transp. Porous Media 107, 1 (2015). doi: 10.1007/s11242-0430-4.

[43] A. Behseresht, A. Noghrehabadi and M. Ghalambaz, Chem. Eng. Res. Des. 92, 3 (2014). doi:10.1016/j.cherd.2013.08.028.

[44] A. Noghrehabadi, M. Ghalambaz and A. Ghanbarzadeh, J. Mech. 30, 3 (2014). http://dx.doi.org/10.1017/jmech.2013.61.

[45] M. Ghalambaz and A. Noghrehabadi, Effects of heat generation/absorption on natural convection of nanofluids over the vertical plate embedded in a porous medium using drift-flux model, J. Comput. Appl. Res. Mech. Eng. Vol. 3, 2 (2014).

[46] B.S. Bhadauria and S. Agarwal, Transp. Porous Media 88, 1 (2011). doi: 10.1007/s11242-0119727-8. 
[47] C. Beckermann, R. Viskanta and S. Ramadhyani, Numer. Heat Transfer 10 (1986). doi: 10.1080/10407788608913535.

$r \quad$ [48] R. Gross, M.R. Bear and C.E. Hickox, in: Proc. $7^{\text {th }}$ IHTC, San Francisco, CA (1986).

\& [49] D.M. Manole and J.L. Lage, Numerical benchmark results for natural convection in a porous - $\quad$ medium cavity, in: Heat Mass Transfer Porous Media, ASME Conference, Vol. 105 (1992).

7 [50] S.L. Moya, E. Ramos and M. Sen, Int. J. Heat Mass Transf. 30 (1987). doi: 10.1016/00179310(87)90204-3.

$\wedge \quad$ [51] A. Bejan, Lett. Heat Mass Transf. 6 (1979). doi: 10.1016/0094-4548(79)90001-8.

9 [52] K.L. Walker, and G.M. Homsy, J. Fluid Mech. 87 (1978). doi: http://dx.doi.org/10.1017/S0022112078001718. 